\title{
Delight and Frustration with Number "Seven" in Plane Geometry and the Regular Heptagon
}

\author{
A. Wünsche \\ Institut für Physik, Humboldt-Universität, Berlin, Germany (Formerly) \\ Email: alfred.wuensche@physik.hu-berlin.de
}

How to cite this paper: Wünsche, A. (2021) Delight and Frustration with Number "Seven" in Plane Geometry and the Regular Heptagon. Advances in Pure Mathematics, 11, 63-100.

https://doi.org/10.4236/apm.2021.111005

Received: November 9, 2020

Accepted: January 25, 2021

Published: January 28, 2021

Copyright (c) 2021 by author(s) and Scientific Research Publishing Inc. This work is licensed under the Creative Commons Attribution International License (CC BY 4.0).

http://creativecommons.org/licenses/by/4.0/ (c) (i) Open Access

\begin{abstract}
As starting point for patterns with seven-fold symmetry, we investigate the basic possibility to construct the regular heptagon by bicompasses and ruler. To cover the whole plane with elements of sevenfold symmetry is only possible by overlaps and (or) gaps between the building stones. Resecting small parts of overlaps and filling gaps between the heptagons, one may come to simple parqueting with only a few kinds of basic tiles related to sevenfold symmetry. This is appropriate for parqueting with a center of seven-fold symmetry that is illustrated by figures. Choosing from the basic patterns with sevenfold symmetry small parts as elementary stripes or elementary cells, one may form by their discrete translation in one or two different directions periodic bordures or tessellation of the whole plane but the sevenfold point-group symmetry of the whole plane is then lost and there remains only such symmetry in small neighborhoods around one or more centers. From periodic tiling, we make the transition to aperiodic tiling of the plane. This is analogous to Penrose tiling which is mostly demonstrated with basic elements of fivefold symmetry and we show that this is also possible with elements of sevenfold symmetry. The two possible regular star-heptagons and a semi-regular star-heptagon play here a basic role.
\end{abstract}

\section{Keywords}

Bicompasses and Ruler Construction, Regular Heptagon, Regular and Semi-Regular Star-Heptagons, Point-Group Symmetry $C_{7}$ and $C_{7 n}$, Parqueting, Tiling, Tessellation, Penrose Tiles, Symmetry and Antisymmetry, Magnetic and Non-Magnetic Classes, Time Inversion, Color Groups

\section{Introduction}

Plane geometry and number theory are considered as the oldest disciplines of mathematics where the historical roots blur in ancient times. Most knowledge 
from ancient mathematics is handed down to modern time by the 13 books of Euclid's "Elements" (for example, Stillwell [1], Maor [2] from the many representations of history of mathematics). Geometric constructions with compass and ruler fascinated professional mathematicians and layman to every time. A particularly interesting special case is constructions of regular polygons ( $n$-gons) and it is known since ancient time that beside the square and the regular triangle $(n=3)$, the regular pentagon $(n=5)$ and their combination to the regular pentadecagon $(n=3.5=15)$ are constructible in such way and, furthermore, all $n$-gons which arise from them by multiple bisections of the central inner angles of the basic isosceles triangles (i.e., $n=3 \cdot 2^{m}, 5 \cdot 2^{m}, 3 \cdot 5 \cdot 2^{m},(m=0,1,2, \cdots)$. The young Gauss found in 1796 that in addition the $n$-gons are constructible by compass and ruler if $n$ is a prime Fermi number $n=F_{h} \equiv 2^{2^{h}}+1 \quad$ (plus possible bisections of the inner angles). From the many possible references we cite here Stillwell [1] because he mentions with citation on p. 512 of the original paper that Wantzel in 1837 finally proved that this is not only sufficient but that it is basically necessary for such constructibility that $n$ is a product of different prime Fermi numbers and completes in this way the insight of Gauss that, apparently, is not very well known up to now (see also Maor and Jost [3], p. 76, for full name and life data of Wantzel). From other possible references, we cite here the very interesting work of Conway and Guy [4] and the nice booklet of Sutton [5]. For $h=2$ one finds the Fermi number $F_{2}=2^{2^{2}}+1=17$ that leads to the famous constructibility of the regular 17-gon, the very new case not known in ancient time. In addition to cases resting on the prime Fermi numbers, clearly, the square and all its cases obtained by bisection of the central inner angles are constructible by compass and ruler (i.e., $\left.n=2 \cdot 2^{m}, m=0,1,2, \cdots\right)$. Formally, the last corresponds to Fermi numbers $F_{h}$ with $h \rightarrow-\infty$ (2-gon or di-gon) but then it is not clear whether or not are there geometric objects which in some sense correspond to the (irrational) Fermi numbers with finite negative integers $-\infty<h<0$.

The $n$-gon with the lowest number $n \geq 3$ which is not constructible by compass and ruler is the regular heptagon to number $n=7$. The prime number "Seven" as symmetry in nature and art is very seldom realized (see Section before "Conclusion") but it plays some role in mystics. We mention here only the very old myth that our world was created in seven days and everybody will find many other things even from daily life which more or less arbitrarily were related by men with the number "Seven".

The regular heptagon can be constructed by a so-called "neusis" construction [4] [5] [6] which is not fully in the spirit of constructions by compass and ruler. It was shown in [7] [8] that a regular heptagon may be constructed by an instrument which was called "rhombic bicompasses" and consists of two compasses which are connected by movable arms of equal lengths (at least three arms altogether) and with fixed endpoints in their action. In this way the points on the two different circles which can be drawn by such an instrument are correlated (see Section 2). It seems to us that such constructions are more in the 
spirit of constructions by compass and ruler than neusis constructions but one has to wait whether or not this will be accepted by the community of mathematicians. In combination with usual compasses the construction of much more regular $n$-gons becomes possible than without it. A technical realization of rhombic bicompasses, in particular with the possibility of variable arm lengths, would be interesting.

The regular heptagon and also the two possible regular star-heptagons possess the point-group symmetry $C_{7 v} \triangleq 7 \mathrm{~m}$ with 14 symmetry elements given in Schoenflies and in International notation ( $m$ stands here for 'mirror' symmetry) used by physicists. This group characterizes a relatively simple symmetry and may serve as starting point for some considerations to extensions. The pure rotation group $C_{7} \wedge 7$ with 7 elements of rotations and the mirror group $C_{s} \equiv C_{1 v}$ are the only possible nontrivial subgroups of $C_{7 V}$ where $C_{7 V}$ may be considered in its structure as built from the subgroup $C_{7}$ of $C_{14}$ where the coset $\left(C_{14}-C_{7}\right)$ to its subgroup $C_{7}$ is multiplied by the "simplest element of antisymmetry" that is the reflection at a line in the plane. This provides the opportunity to consider groups with and without general anti-symmetries which became important in physics, for example, as magnetic groups and non-magnetic groups if this element of anti-symmetry is the inversion of time. However, the extensions of the point groups $C_{7}$ or $C_{7 V}$ to crystallographic groups is as it is well known not possible that restricts their possibilities. Furthermore, one may discuss possible realizations of $C_{7 V}$ and $C_{7}$ for parqueting and tessellations of planes and this goes in direction of one- and two-dimensional reliefs and tiling and art-work and also to realizations in nature.

The number of contributions to symmetry and to discrete groups and of their extension to anti-symmetries and color groups and to application in physics, in particular, in crystallography is really enormous and their notations are often very different in physics and mathematics. An early classical book about symmetries in art and nature is that of $\mathrm{H}$. Weyl [9] ${ }^{1}$ and later to parts the book of Steinhaus [10]. One of my earliest books about regular and semi-regular symmetries was that of Lyusternik [11] with general propositions about convex figures and with the regular and semi-regular Archimedean polyhedrons. The more extensive book of Fejes Tóth [12] contains also mathematical propositions to two-dimensional figures and three-dimensional objects and in addition good-quality reproductions to plane figures and red-green stereo-spectacles for spatially seeing three-dimensional geometrical objects.

Next, we have to mention the Russian school (it seems that one may speak about such) with roots, in particular, from E. S. Fyodorov who in 1890 (and independently Schoenflies in 1891) found the crystallographic spatial groups and later from Shubnikov who contributed to symmetries and anti-symmetries with applications in physics where they play an important rule (e.g., [13]). The most important of the many journal papers of Shubnikov are collected in the work [14] ${ }^{1}$ Translations of books from Western countries into Russian were welcome since up to the turn in GDR in 1990 it was impossible for me to buy them in original. 
which comprises as well physical as also mathematical contributions to symmetry groups with particularly beautiful illustrations to them and tabular material. Anti-symmetry in mathematical form was first considered by $\mathrm{H}$. Heesch and as time inversion in addition to spatial crystallographic symmetry elements by Shubnikov, Koptsik and others and leads to magnetic and non-magnetic point-groups and crystal classes. A very broad spectrum of ideas to theory and application of discrete symmetries is dealt with in the book of Shubnikov and Koptsik [15] with many tables and symbolic representations (in black-red for anti-symmetries and by more colors for color groups) and also in nature with (black-white) reproductions of E. Haeckel and artwork is also taken into account. More for specialists and very astonishing is the large monograph of Koptsik [16] with symbolic graphical representation of all 90 generalized point groups and of all $1421=230+1191$ generalized crystallographic groups (in black-red and with many tabular material). In Russian literature the usual 230 crystallographic groups are mostly called "Fyodorov groups", with antisymmetry "Shubnikov groups" and with elements of more general color symmetry "Byelov groups". About magnetic crystal classes in physics we recommend also the paragraphs $\$ 57$ and $\$ 58$ of [17] in connection with the very well-written chap. XII to symmetry of Landau and Lifshits [18]. A special article about magnetic crystals is that of Dimmock and Wheeler [19]. To history of crystallography and, more generally, of geometry, we find very interesting and little known stories in the popular-scientific booklet of Levitin $[20]^{2}$.

A well organized representation of group theory for physicists and mathematicians together with vast tabular material to the crystallographic point and space groups in the Appendices we find in the monograph of Lyubarskiy [21]. From the more physically oriented representations of the mathematics of symmetry in application to crystallography and the notations we recommend the book of Yale [22] and, in particular, the first chapter (about 80 pages) of the monograph of Kleber [23].

In our paper [24] we gave the structure of the 122 generalized crystallographic point groups built from the 11 crystallographic point groups with only rotations as elements of symmetry $\left(C_{1}, C_{2}, D_{2}, C_{3}, D_{3}, C_{4}, D_{4}, C_{6}, D_{6}, T, O\right)$ from their non-trivial 10 subgroups of divisor 2 and among them 3 of divisor 4 by multiplication of their co-sets by the elements of $\overline{1}$ (spatial inversion), 1 (time inversion) and by $\overline{1}$ (product of spatial inversion and time inversion) and by direct products with them that leads to a good overview about them and to magnetic and non-magnetic and to gyrotropic and non-gyrotropic classes.

From a more mathematical point of view to symmetry of two-, three- and higher-dimensional geometrical objects is the work of Coxeter, e.g. [25]. An al-

${ }^{2}$ For example, it is said that E. S. Fyodorov found 229 and Schoenflies 227 of the possible 230 crystallographic space groups without intersection of the absent groups that means the union of both works contains all crystallographic space groups. There is also reported that the derivation of the 17 planar net (or lattice) groups which can be extended by translation from the appropriate 10 planar point groups to two-dimensional analogs of three-dimensional crystallographic groups and which often is attributed to Pólya was already made before by E. S. Fyodorov ([20], chap. V, pp. 90 and 80). 
most exhaustable representation of two-dimensional symmetries for tilings and tessellations with a great number of high-quality figures (in black-white) one finds in the voluminous monograph of Grünbaum and Shephard [26]. Computers make it possible to create now really beautiful colored geometrical figures. A very novel representation with many new ideas (e.g., costs of patterns, magic theorem) and new notations for extended considerations of symmetries and with a great number of two- and three-dimensional figures and with explanation of their symmetries is given in the book of Conway, Burgiel and Goodman-Strauss [27]. Also with beautiful symmetrical figures but without mathematics and more from an artists point of view is the work of Miyazaki [28]. Likely, there are much more similar sources which had to be cited.

With figures of arbitrary point-group symmetry one may fill the whole plane but only a few point-group symmetries are compatible with additional translation symmetry. In three-dimensional case these are the well-known 7 crystal systems in the possible 14 Bravais lattices with 32 possible point groups and 230 "usual" crystallographic space groups. In the two-dimensional case these are analogously 5 lattice systems with 10 point-group symmetries and 17 possible net groups as analogues of the crystallographic space groups [15] [26] [27]. All point-group symmetries with number five and equal and higher of seven are not possible in connection with discrete translation symmetries. The covering of spaces by geometrical objects (for examples, by circles, balls and analogously $n$-dimensional spheres in $n$-dimensional spaces) including such with overlaps are considered in the highly mathematical book of Conway and Sloane [29]. Covering of the whole plane with heptagons with small overlapping and after their cut-off with a very small number of remaining different tiles are possible and considered in present article. A similar and very interesting direction was initiated already long ago by Penrose (in about 1976) and found interesting applications in quasi-crystals. Two introducing articles from Martin Gardner we find in [30] (first two chapters pp. 1-30) with beautiful figures (in particularly impressive for me are Figure 8, Figure 9 and Figure 11 of patterns "sun", "star" and "cartwheel" and the Ammann bars) and more in the already cited monograph of Grünbaum and Shephard [26] with a voluminous chapter about aperiodic tilings (chap. 10, pp. 519-582) including a large number of pretty pictures. Practically all such patterns in the cited sources contain locally elements of five-fold symmetry (regular pentagon and star-pentagon and a semi-regular pentagon) and the gaps between them are filled with other simple polygons. It seems to us that aperiodic tiling of the kind of Penrose tiles is also possible with elements of local seven-fold symmetry such as the regular heptagon and the two regular star-heptagons and some figures were made for present article and for preparation in this direction. This direction is very open for further investigation.

\section{An Ancient Theorem for a Doubling Relation between Two Angles within a Circle}

In this short Section we give a theorem known from ancient time for a doubling 
relation between two angles constructed within a circle. It is very useful for quickly establishing relations between the different angles inside and outside of regular polygons. According to Maor [2] (chap. 6) it is Proposition 20 and Proposition 21 of book III of Euclid's “Elements". Probably, it is much older and was known already to the Babylonians. The Figs. 28-33 in [2] illustrate what are equivalent contents of the theorem. In the book of Stillwell [1] this theorem is almost at the beginning of the whole representation (p. 8, Fig. 1.6). Nevertheless, it seems to be not so well-known and popular as, for example, the theorem of Pythagoras.

The theorem is illustrated for our purpose in Figure 1. Its geometrical proof is very simple but it has to use the theorem that the angle sum of an arbitrary plane triangle is equal to $\pi$ that is also easily to prove geometrically, for example, by fragmentation of two equal triangles and compositions of the fragments to a rectangle. The triangle with corners $(O, B, C)$ is an isosceles one and therefore the inner angles at $B$ and $C$ are equal and are denoted by $\alpha$. Due to the angle sum of a triangle the inner angle at $O$ is equal to $\pi-2 \alpha$ and the complementary angle to it denoted by $\beta$ is equal to $2 \alpha$. The triangle with the corners $(O, A, B)$ is also an isosceles one and therefore its inner angles at points $A$ and $B$ are equal and due to the angle sum within a triangle are then equal to $\frac{\pi}{2}-\alpha$. This proves at once that the inner angle of the triangle with the corners $(A, B, C)$ at point $B$ is a right angle.

A modern analytic proof of the doubling theorem is easily to make using the theorem of Pythagoras and the analytic form of doubling relations for the angles in trigonometric functions, for example, for the Tangent but due to clearness of the geometrical proof it is not necessary to give it here. For the relations between

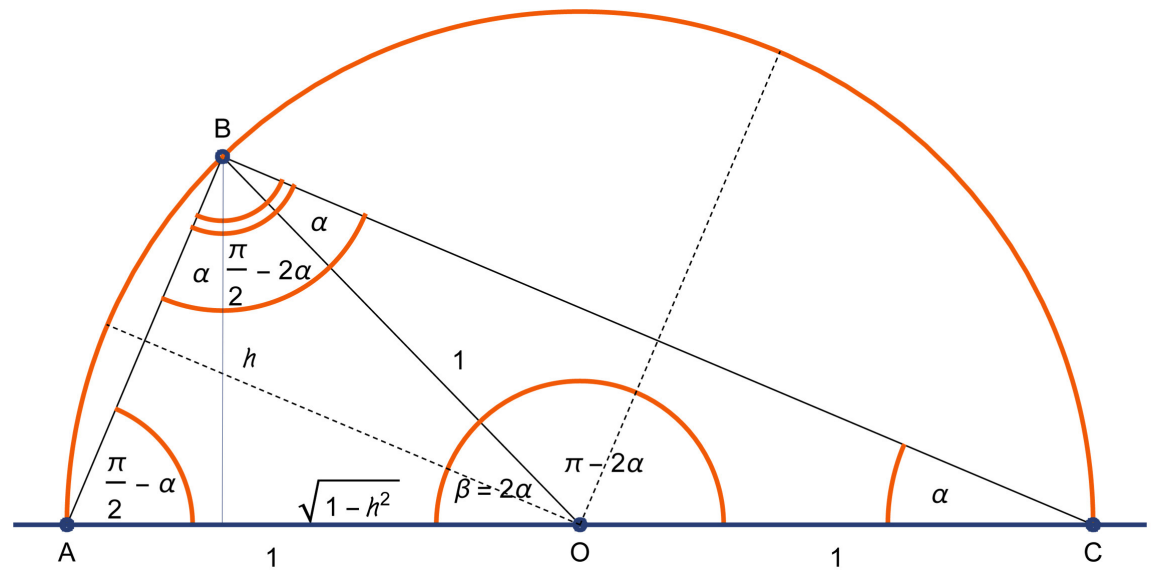

Figure 1. Doubling relation between two angles $\alpha$ and $\beta=2 \alpha$ within a (unit) circle. The triangles with the corners $(O, A, B)$ and $(O, B, C)$ are isosceles ones and the triangle with corners $(A, B, C)$ is a rectangular one. The elementary proof uses the theorem that the angle sum of arbitrary plane triangles is equal to $\pi$. The theorem extends from acute to obtuse angles $\beta$. (Figure such as all following figures made by "Mathematica 10"). 
the angles of regular polygons it is convenient to use the doubling theorem but it is also possible by other reasoning. In last cases it should implicitly contain a proof of the doubling theorem without seeing this.

\section{Cyclotomic Equation and Its Solution for the Regular Heptagon}

The corner points of a regular $n$-gon inscribed into a circle of unit radius $(R=1)$ and considered in the complex $z$-plane is the cyclotomic equation

$$
0=z^{n}-1=(z-1)\left(z^{n-1}+z^{n-2}+\cdots+z+1\right), \quad(z=x+i y) .
$$

Their $n$ solutions by, in general, transcendental numbers $z_{k}$ (modulo $n$ ) are

$$
\begin{gathered}
z_{k}=\exp \left(i k \frac{2 \pi}{n}\right)=\cos \left(k \frac{2 \pi}{n}\right)+i \sin \left(k \frac{2 \pi}{n}\right), \quad(k=0,1,2, \cdots, n-1), \\
z_{0}=z_{7}=1, \quad z_{k} z_{l}=z_{k+1}, \quad z_{k}=z_{n-k}^{*}, \quad\left|z_{k}\right| \equiv \sqrt{z_{k} z_{k}^{*}}=1 .
\end{gathered}
$$

They are constructible by compass and ruler if these solutions do not involve irrational expressions higher than quadratic radicals (including nested quadratic radicals). For the construction by rhombic bicompasses and ruler this has to be weakened by the requirement that all fixed points used for the construction should be determined by irrational expressions not more complicated than nested quadratic radicals.

The cyclotomic equation for the regular heptagon can be factorized as follows [7] [8]

$$
\begin{aligned}
0 & =z^{7}-1 \\
& =(z-1)\left(z^{6}+z^{5}+z^{4}+z^{3}+z^{2}+z+1\right) \\
& =(z-1)\left(z^{3}+\frac{1-i \sqrt{7}}{2} z^{2}-\frac{1+i \sqrt{7}}{2} z-1\right)\left(z^{3}+\frac{1+\mathrm{i} \sqrt{7}}{2} z^{2}-\frac{1-\mathrm{i} \sqrt{7}}{2} z-1\right) \\
& =(z-1)\left(\left(z-z_{1}\right)\left(z-z_{2}\right)\left(z-z_{4}\right)\right)\left(\left(z-z_{3}\right)\left(z-z_{5}\right)\left(z-z_{6}\right)\right)
\end{aligned}
$$

It is easily seen that the first polynomial in $z$ of third degree involves the three roots $z_{1}, z_{2}, z_{4}$ and the second polynomial of third degree the three roots $z_{3}, z_{5}, z_{6}$ and due to the theorem of Viète we have (Figure 2)

$$
\begin{gathered}
z_{1}+z_{2}+z_{4}=z_{3} z_{5}+z_{3} z_{6}+z_{5} z_{6}=\frac{-1+i \sqrt{7}}{2}, \\
z_{3}+z_{5}+z_{6}=z_{1} z_{2}+z_{1} z_{4}+z_{2} z_{4}=\frac{-1-i \sqrt{7}}{2}, \\
z_{1} z_{2} z_{4}=z_{7}=z_{3} z_{5} z_{6}=z_{14}=z_{0}=1 .
\end{gathered}
$$

For convenience let us give the solutions also in numerical form. The seven-th complex roots of 1 are

$$
\begin{aligned}
& z_{0}=z_{7}=1 \\
& z_{1}=\cos \left(\frac{2 \pi}{7}\right)+i \sin \left(\frac{2 \pi}{7}\right)=+0.6234898019+i 0.7818314825,
\end{aligned}
$$




$$
\begin{aligned}
& z_{2}=\cos \left(\frac{4 \pi}{7}\right)+i \sin \left(\frac{4 \pi}{7}\right)=-0.2225209340+i 0.9749279122 \\
& z_{3}=\cos \left(\frac{6 \pi}{7}\right)+i \sin \left(\frac{6 \pi}{7}\right)=-0.9009688679+i 0.4338837391 \\
& z_{4}=\cos \left(\frac{8 \pi}{7}\right)+i \sin \left(\frac{8 \pi}{7}\right)=-0.9009688679-i 0.4338837391 \\
& Z_{5}=\cos \left(\frac{10 \pi}{7}\right)+i \sin \left(\frac{10 \pi}{7}\right)=-0.2225209340-i 0.9749279122 \\
& z_{6}=\cos \left(\frac{12 \pi}{7}\right)+i \sin \left(\frac{12 \pi}{7}\right)=+0.6234898019-i 0.7818314825
\end{aligned}
$$

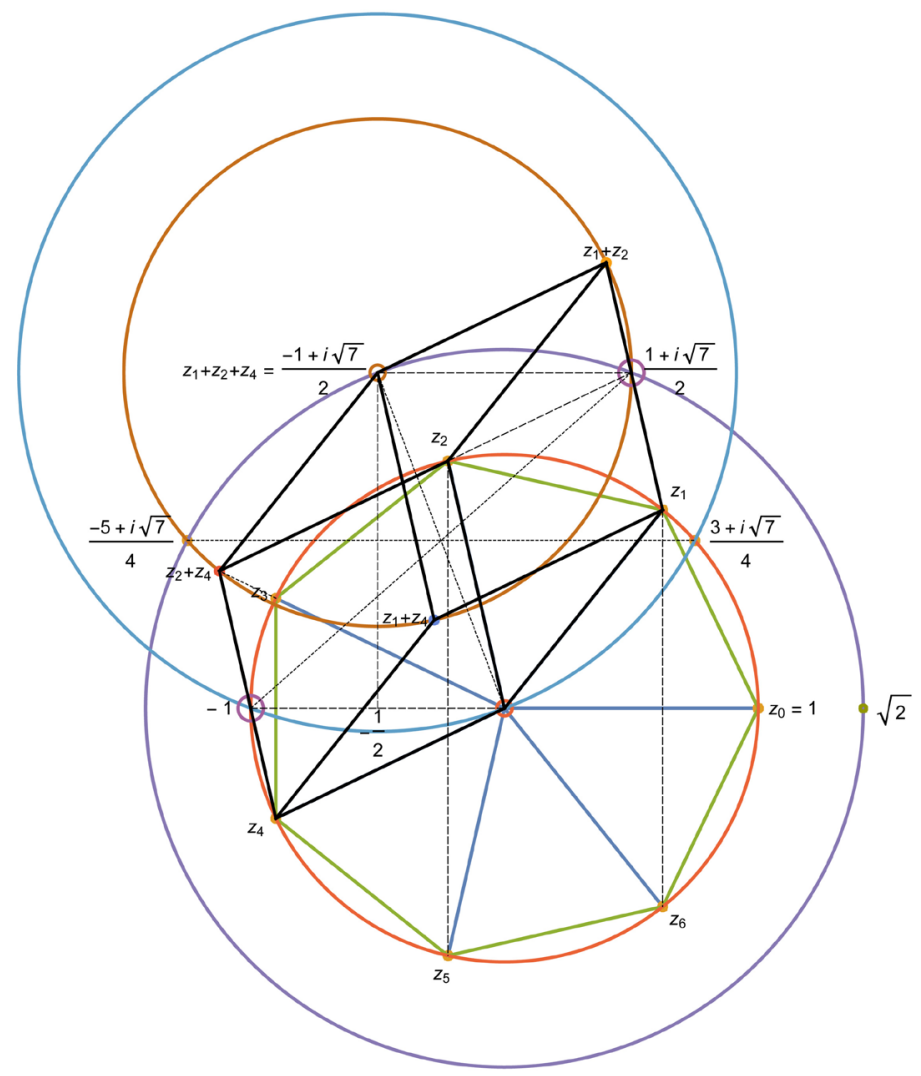

Figure 2. Regular heptagon and its construction with rhombic bicompasses and ruler in complex $z$-plane. Additionally to the fixed points for the bicompasses and corresponding circles of radius $R_{1}=1$ we have drawn two circles with Radius $R_{2}=\sqrt{2}$ around the coordinate origin and around the point $\frac{-1+i \sqrt{7}}{2}$ which are here the fixed points for the rhombic bicompasses with distance $\sqrt{2}$ and obtain in this way a modified construction by absolute minimum bicompasses with 3 arms. For the construction it is important that the middle arm of the bicompasses goes through the point $\frac{1+i \sqrt{7}}{2}$ or through the point -1 in the complex plane. The complex points $z_{3}$ and $z_{2}+z_{4}=2 \cos \left(\frac{2 \pi}{7}\right) z_{3}=1.246980 z_{3}$ lie on the same straight line from the center. 
They are the corner-points of the regular heptagon in the complex plane inscribed into a circle of radius equal to $R=1$.

\section{Equations for Real and Imaginary Part of Roots for the Regular Heptagon}

It is interesting to establish the equations of third degree which provide the real and imaginary parts $x_{k}$ and $y_{k}$ of the solutions $z_{k}$ separately. It is not possible to make this directly by elimination of one part from the root theorem of Viète (3.4) because this provides polynomial equations of nine-th degree. This is due to the fact that the elimination process does not know which are the pairs of real and imaginary part of the solutions and takes into account all possible combinations.

For the sums it follows directly from the first of the relations (3.4) that

$$
\begin{gathered}
x_{1}+x_{2}+x_{4}=x_{6}+x_{5}+x_{3}=\cos \left(\frac{2 \pi}{7}\right)+\cos \left(\frac{4 \pi}{7}\right)+\cos \left(\frac{8 \pi}{7}\right)=-\frac{1}{2}, \\
y_{1}+y_{2}+y_{4}=-y_{6}-y_{5}-y_{3}=\sin \left(\frac{2 \pi}{7}\right)+\sin \left(\frac{4 \pi}{7}\right)+\sin \left(\frac{8 \pi}{7}\right)=\frac{\sqrt{7}}{2} .
\end{gathered}
$$

Both these identities cannot be obtained by the addition theorems for trigonometric functions alone and have to use the theorem of Viète. Using well-known identity relations for products of trigonometric functions we may transform the following sums to the sums in (4.1)

$$
\begin{aligned}
& x_{1} x_{2}+x_{1} x_{4}+x_{2} x_{4}=x_{6} x_{5}+x_{6} x_{3}+x_{5} x_{3} \\
&=\cos \left(\frac{2 \pi}{7}\right)+\cos \left(\frac{4 \pi}{7}\right)+\cos \left(\frac{8 \pi}{7}\right)=-\frac{1}{2}, \\
& y_{1} y_{2}+y_{1} y_{4}+y_{2} y_{4}=y_{6} y_{5}+y_{6} y_{3}+y_{5} y_{3}=0,
\end{aligned}
$$

and, furthermore, using trigonometric identities and (4.1)

$$
\begin{gathered}
x_{1} x_{2} x_{4}=x_{6} x_{5} x_{3}=\frac{1}{4}\left\{1+\cos \left(\frac{2 \pi}{7}\right)+\cos \left(\frac{4 \pi}{7}\right)+\cos \left(\frac{8 \pi}{7}\right)\right\}=\frac{1}{8}, \\
y_{1} y_{2} y_{4}=-y_{6} y_{5} y_{3}=-\frac{1}{4}\left\{\sin \left(\frac{2 \pi}{7}\right)+\sin \left(\frac{4 \pi}{7}\right)+\sin \left(\frac{8 \pi}{7}\right)\right\}=-\frac{\sqrt{7}}{8} .
\end{gathered}
$$

Thus the equation which provides the real parts of the complex roots as solutions is

$$
\begin{aligned}
0 & =x^{3}+\frac{1}{2} x^{2}-\frac{1}{2} x-\frac{1}{8} \\
& =\left(x-x_{1}\right)\left(x-x_{2}\right)\left(x-x_{4}\right)=\left(x-x_{6}\right)\left(x-x_{5}\right)\left(x-x_{3}\right),
\end{aligned}
$$

and the equation for the imaginary parts of the complex roots

$$
\begin{aligned}
0 & =y^{3}-\frac{\sqrt{7}}{2} y^{2}+\frac{\sqrt{7}}{8} \\
& =\left(y-y_{1}\right)\left(y-y_{2}\right)\left(y-y_{4}\right)=\left(y+y_{6}\right)\left(y+y_{5}\right)\left(y+y_{3}\right) .
\end{aligned}
$$

The coefficients in the equations for the real and the imaginary part of the 
complex roots are only rational numbers or rational parts of the square root of 7 . Their parts in the Cardano formulae, however, are complex numbers and are rather complicated.

If we denote the solutions for the doubled imaginary part of the corner-points by $u_{k}$ of the regular heptagon according to

$$
u_{k} \equiv 2 y_{k}=2 \sin \left(k \frac{2 \pi}{7}\right), \quad(k=0,1,2, \cdots, 6),
$$

then we find from (4.5) the two equations for the doubled imaginary parts

$$
\begin{gathered}
0=u^{3}-\sqrt{7} u^{2}+\sqrt{7}=\left(u-u_{1}\right)\left(u-u_{2}\right)\left(u-u_{4}\right), \\
0=u^{3}+\sqrt{7} u^{2}-\sqrt{7}=\left(u-u_{6}\right)\left(u-u_{5}\right)\left(u-u_{3}\right) .
\end{gathered}
$$

For the product of both equations we get the following bi-cubic equation for the quantity $u$

$$
0=\left(u^{3}-\sqrt{7} u^{2}+\sqrt{7}\right)\left(u^{3}+\sqrt{7} u^{2}-\sqrt{7}\right)=u^{6}-7 u^{4}+14 u^{2}-7 .
$$

In last form of the right-hand side this is the bi-cubic equation of Kepler with integer coefficients (see Fejes Tóth [12], p. 117) for the side lengths of the regular heptagon $\{7 / 1\}$, the first regular star-heptagon $\{7 / 2\}$ and the second regular star-heptagon $\{7 / 3\}$ all inscribed with their corners into a circle of radius $R=1$. The applied symbols for regular heptagon and regular star-heptagons are the Schläfli symbols [12] [25] [27]. The Equation (4.8) possesses only real solutions but they are pair-wise positive and negative ones and the mentioned side lengths mean the positive ones.

\section{Proof for Second Fix-Point of Rhombic Bicompasses Leading to Corner-Points of Regular Heptagon}

We now come to the explanation of the construction of the roots (corners of regular heptagon) (3.5) of the cyclotomic Equation (3.3) by bicompasses and ruler. If we draw the sum of the vectors $\left(z_{1}, z_{2}, z_{4}\right)$ in different order we get a rhombus which ends at the point $\frac{-1+\mathrm{i} \sqrt{7}}{2}$ of the complex plane if we begin in the center of the complex plane. This is shown in Figure 2 where on the circle $z z^{*}=x^{2}+y^{2}=1$ lie all sevenths roots of 1 and on the circle $\left(x+\frac{1}{2}\right)^{2}+\left(y-\frac{\sqrt{7}}{2}\right)^{2}=1$ the three root sums $\left(z_{1}+z_{2}, z_{1}+z_{4}, z_{2}+z_{4}\right)$; (the root sums $\left(z_{3}+z_{5}, z_{3}+z_{6}, z_{5}+z_{6}\right)$ lie on the here not drawn complex conjugated circle in the lower half-plane). The rhombic bicompasses with at least three linearly connected arms of equal lengths is fixed in two points, first in the coordinate origin $z=0$ and second in the point $z=\frac{-1+i \sqrt{7}}{2}$ and the movable arms with one degree of freedom possess a position where they are at once correlated between the points $\left(z_{1}, z_{2}, z_{4}\right)$ of the unit circle around the origin. The two auxiliary points are determined as points of intersection of the two unit cir- 
cles with two circles of radius $\sqrt{2}$ around the same fixed points $z=0$ and $z=\frac{-1+\mathrm{i} \sqrt{7}}{2}$. The square root $\sqrt{2}$ is the distance of the two fixed points of the bicompasses. One of the possible positions of the bicompasses corresponds then to the right corners of the regular heptagon. To find these points one may use one of the two auxiliary points of the complex plane $z=-1$ or $z=\frac{1+i \sqrt{7}}{2}$ at which one of the arms of the rhombic bicompasses intersects these points as shown in Figure 2. We now prove that this determines the right position of the rhombic bicompasses and choose for this purpose the auxiliary point $z=-1$.

The point $z=-1$ lies on the line between $z_{4}$ and $z_{2}+z_{4}$ which can be parameterized by

$$
z=t z_{2}+z_{4}, \quad(0 \leq t \leq 1)
$$

with real parameter $t$. It goes for $t=0$ through the point $z_{4}$ and for $t=1$ through the point $z_{2}+z_{4}$. From the equation

$$
z=t z_{2}+z_{4}=-1,
$$

follows that this is the case for the real value of the parameter $t$ equal to

$$
t=-\frac{z_{4}+1}{z_{2}}=-\left(z_{2}+z_{2}^{*}\right)=-2 \cos \left(\frac{4 \pi}{7}\right)=0.445042 .
$$

The point $z_{2}+z_{4}$ is on the prolongation of the line from the coordinate origin to the point $z_{3}$ according to

$$
\begin{aligned}
z_{2}+z_{4} & =\exp \left(i \frac{4 \pi}{7}\right)+\exp \left(i \frac{8 \pi}{7}\right)=\left\{\exp \left(-i \frac{2 \pi}{7}\right)+\exp \left(i \frac{2 \pi}{7}\right)\right\} \exp \left(i \frac{6 \pi}{7}\right) \\
& =2 \cos \left(\frac{2 \pi}{7}\right) z_{3}=1.246980 z_{3} .
\end{aligned}
$$

This proves that in the right position when a middle arm of the bicompasses intersects the point $z=-1$ it determines the corners $\left(z_{1}, z_{2}, z_{4}\right)$ of the regular heptagon. Analogous considerations can be made for the intersection point $z=\frac{1+i \sqrt{7}}{2}$. If we use only 3 arms of the rhombic bicompasses we may determine by this construction only the point $z_{4}$. By succeeding angle bisection one may determine next the point $z_{2}$ and then finally the points $z_{1}$ and $z_{3}$ and thus also $z_{6}$ and $z_{5}$.

The second fix-point $z=\frac{-1+\mathrm{i} \sqrt{7}}{2}$ for the construction of the regular heptagon with rhombic bicompasses is equal to a second possible basis besides the basis equal to 1 of the Kleinian lattice that means one of the 9 possible lattices with unique factorization [4] [31]. However, it seems now that this is likely only a curious fact with no relation between each other and besides the basis $\left(1, \frac{-1+i \sqrt{7}}{2}\right)$ of the Kleinian lattice of the elementary cell one may choose infi- 
nitely many others, e.g., $\left(\frac{1+i \sqrt{7}}{2}, \pm \frac{-1+i \sqrt{7}}{2}\right)$. Therefore the point-group symmetry of the Kleinian prime integers should possess the $x$ - and $y$-axis as mirror lines and should be not higher than $C_{2 v}$ and lattices with translation symmetries are not compatible with sevenfold symmetry.

The regular heptagon loses a little its fear in view of the first regular $n$-gon which is not constructible by compass and ruler between the cases of the regular trigon $n=3$ and the regular octagon $n=8$ since it is constructible instead by rhombic bicompasses and ruler.

\section{Geometry of the Regular Heptagon and Role of Addition and Multiplication of Complex Numbers for Two-Dimensional Rotations and Translations}

For the drawings of figures in the next sections it is steadily necessary to make rotations of points and lines. The rotation of plane coordinates $r=\left(\begin{array}{l}x \\ y\end{array}\right)$ about an angle $\varphi$ counter-clockwise around the coordinate origin (transformation $\mathrm{R}(\varphi))$ with following translation by a vector $\boldsymbol{r}_{0}=\left(\begin{array}{l}x_{0} \\ y_{0}\end{array}\right)$ is represented by

$$
\begin{gathered}
\boldsymbol{r}^{\prime}=\mathrm{R}(\varphi) \boldsymbol{r}+\boldsymbol{r}_{0}, \\
\left(\begin{array}{c}
x^{\prime} \\
y^{\prime}
\end{array}\right)=\left(\begin{array}{rr}
\cos (\varphi) & -\sin (\varphi) \\
\sin (\varphi) & \cos (\varphi)
\end{array}\right)\left(\begin{array}{l}
x \\
y
\end{array}\right)+\left(\begin{array}{l}
x_{0} \\
y_{0}
\end{array}\right)=\left(\begin{array}{c}
\cos (\varphi) x-\sin (\varphi) y+x_{0} \\
\sin (\varphi) x+\cos (\varphi) y+y_{0}
\end{array}\right) .
\end{gathered}
$$

Alternatively, this is exactly what is made with the algebra of complex numbers $z=x+i y$ by addition and multiplication with complex numbers of modulus equal to 1

$$
\begin{aligned}
z^{\prime} & =x^{\prime}+\mathrm{i} y^{\prime}=\exp (\mathrm{i} \varphi) z+z_{0} \\
& =(\cos (\varphi)+\mathrm{i} \sin (\varphi))(x+\mathrm{i} y)+\left(x_{0}+\mathrm{i} y_{0}\right) \\
& =\cos (\varphi) x-\sin (\varphi) y+x_{0}+\mathrm{i}\left(\sin (\varphi) x+\cos (\varphi) y+y_{0}\right)
\end{aligned}
$$

In this principal way we made the calculations to the group of Euclidean motions for the drawings, however, mostly in other ordering, first the translation from the coordinate origin and then the rotations of the transformed vectors or lines or geometrical objects

$$
\boldsymbol{r}^{\prime \prime}=\mathrm{R}(\varphi)\left(\boldsymbol{r}+\boldsymbol{r}_{0}\right)=\boldsymbol{r}^{\prime}+(\mathrm{R}(\varphi)-\mathrm{I}) \boldsymbol{r}_{0} .
$$

The algebra of complex numbers is essentially the algebra of the two-dimensional rotation group $S O(2)$ which extended by multiplication with real numbers unequal to zero is the maximal group of continuous commutative transformations with a fixed center and already the two-dimensional unitary unimodular group $S U(2)$ with 3 independent parameters is non-commutative. For the description of the transformations by such non-commutative groups was searched for hyper-complex algebraic number systems (e.g., [31]) which may 
describe them (e.g., Hamilton"s quaternions) but the general form of their description by matrices (or linear operators) was soon later developed by Cayley and others.

The distance $D\left(z_{1}, z_{2}\right)$ of two complex numbers $z_{1}=r_{1} \mathrm{e}^{\mathrm{i} \varphi_{1}}$ and $z_{2}=r_{2} \mathrm{e}^{\mathrm{i} \varphi_{2}}$ or equivalent plane vectors $\boldsymbol{r}_{1}$ and $\boldsymbol{r}_{2}$ is determined by

$$
\begin{aligned}
D\left(z_{1}, z_{2}\right) & =\sqrt{\left(z_{1}-z_{2}\right)\left(z_{1}^{*}-z_{2}^{*}\right)} \\
& =\sqrt{x_{1}^{2}+y_{1}^{2}+x_{2}^{2}+y_{2}^{2}-2\left(x_{1} x_{2}+y_{1} y_{2}\right)} \\
& =\sqrt{\boldsymbol{r}_{1}^{2}+\boldsymbol{r}_{2}^{2}-2 \boldsymbol{r}_{1} \boldsymbol{r}_{2}} .
\end{aligned}
$$

The Cosine and Sine between two complex numbers $z_{1}$ and $z_{2}$ are determined by

$$
\begin{gathered}
\cos \left(\varphi_{1}-\varphi_{2}\right)=\frac{z_{1} z_{2}^{*}+z_{1}^{*} z_{2}}{2 \sqrt{\left(z_{1} z_{1}^{*}\right)\left(z_{2} z_{2}^{*}\right)}}=\frac{x_{1} x_{2}+y_{1} y_{2}}{\sqrt{\left(x_{1}^{2}+y_{1}^{2}\right)\left(x_{2}^{2}+y_{2}^{2}\right)}}=\frac{\boldsymbol{r}_{1} \boldsymbol{r}_{2}}{\left|\boldsymbol{r}_{1}\right|\left|\boldsymbol{r}_{2}\right|}, \\
\sin \left(\varphi_{1}-\varphi_{2}\right)=-\mathrm{i} \frac{z_{1} z_{2}^{*}-z_{1}^{*} z_{2}}{2 \sqrt{\left(z_{1} z_{1}^{*}\right)\left(z_{2} z_{2}^{*}\right)}}=\frac{y_{1} x_{2}-x_{1} y_{2}}{\sqrt{\left(x_{1}^{2}+y_{1}^{2}\right)\left(x_{2}^{2}+y_{2}^{2}\right)}} \equiv \frac{\left[\boldsymbol{r}_{1}, \boldsymbol{r}_{2}\right]}{\left|\boldsymbol{r}_{1}\right|\left|\boldsymbol{r}_{2}\right|},
\end{gathered}
$$

with $[\boldsymbol{a}, \boldsymbol{b}]$ the vector product of vector $\boldsymbol{a}$ with vector $\boldsymbol{b}$.

Some features of the geometry of the regular heptagon with circumscribed circle of radius $R=1$ are represented in Figure 3. The side length $a$ of the regular heptagon and the height $h$ (perpendicular bisector) of the central sector with inner angle $\alpha_{2}=\frac{2 \pi}{7}$ are

$$
a=2 \sin \left(\frac{\pi}{7}\right)=0.867767, \quad h=\cos \left(\frac{\pi}{7}\right)=0.900969 .
$$

The height $h$ is at once the radius of the circle inscribed into the regular heptagon (not drawn in Figure 3). The points on the $x$-axis in Figure 3 are at

$$
\begin{aligned}
A & =\left(\frac{z_{3}+z_{4}}{2}, 0\right)=\left(\cos \left(\frac{6 \pi}{7}\right), 0\right)=(-0.900970,0), \\
B & =\left(-\frac{z_{2}-z_{5}}{z_{2}-z_{5}+z_{3}-z_{4}}, 0\right)=\left(-\frac{\sin \left(\frac{4 \pi}{7}\right)}{\sin \left(\frac{4 \pi}{7}\right)+\sin \left(\frac{6 \pi}{7}\right)}, 0\right)=\left(\frac{\cos \left(\frac{5 \pi}{7}\right)}{\cos \left(\frac{\pi}{7}\right)}, 0\right) \\
& =\left(2 \cos \left(\frac{4 \pi}{7}\right)-2 \cos \left(\frac{2 \pi}{7}\right)+1,0\right)=(-0.692021,0), \\
C & =\left(-\frac{z_{3}-z_{4}}{z_{1}-z_{6}+z_{3}-z_{4}}, 0\right)=\left(-\frac{\sin \left(\frac{6 \pi}{7}\right)}{\sin \left(\frac{6 \pi}{7}\right)+\sin \left(\frac{2 \pi}{7}\right)}, 0\right) \\
& =\left(\frac{\cos \left(\frac{4 \pi}{7}\right)}{\cos \left(\frac{2 \pi}{7}\right)}, 0\right)=\left(-\frac{\sin \left(\frac{\pi}{14}\right)}{\sin \left(\frac{3 \pi}{14}\right)}, 0\right)=(-0.356896,0),
\end{aligned}
$$




$$
\begin{aligned}
D & =\left(\frac{z_{2}+z_{5}}{2}, 0\right)=\left(\cos \left(\frac{4 \pi}{7}\right), 0\right)=(-0.222521,0), \\
E & =\left(\frac{z_{3}-z_{4}}{z_{1}-z_{6}+z_{2}-z_{5}}, 0\right)=\left(+\frac{\sin \left(\frac{6 \pi}{7}\right)}{\sin \left(\frac{2 \pi}{7}\right)+\sin \left(\frac{4 \pi}{7}\right)}, 0\right) \\
& =\left(\frac{\cos \left(\frac{3 \pi}{7}\right)}{\cos \left(\frac{\pi}{7}\right)}, 0\right)=\left(2 \cos \left(\frac{2 \pi}{7}\right)-1,0\right)=(+0.246980,0), \\
F & =\left(\frac{z_{1}+z_{6}}{2}, 0\right)=\left(\cos \left(\frac{2 \pi}{7}\right), 0\right)=(+0.623490,0) .
\end{aligned}
$$

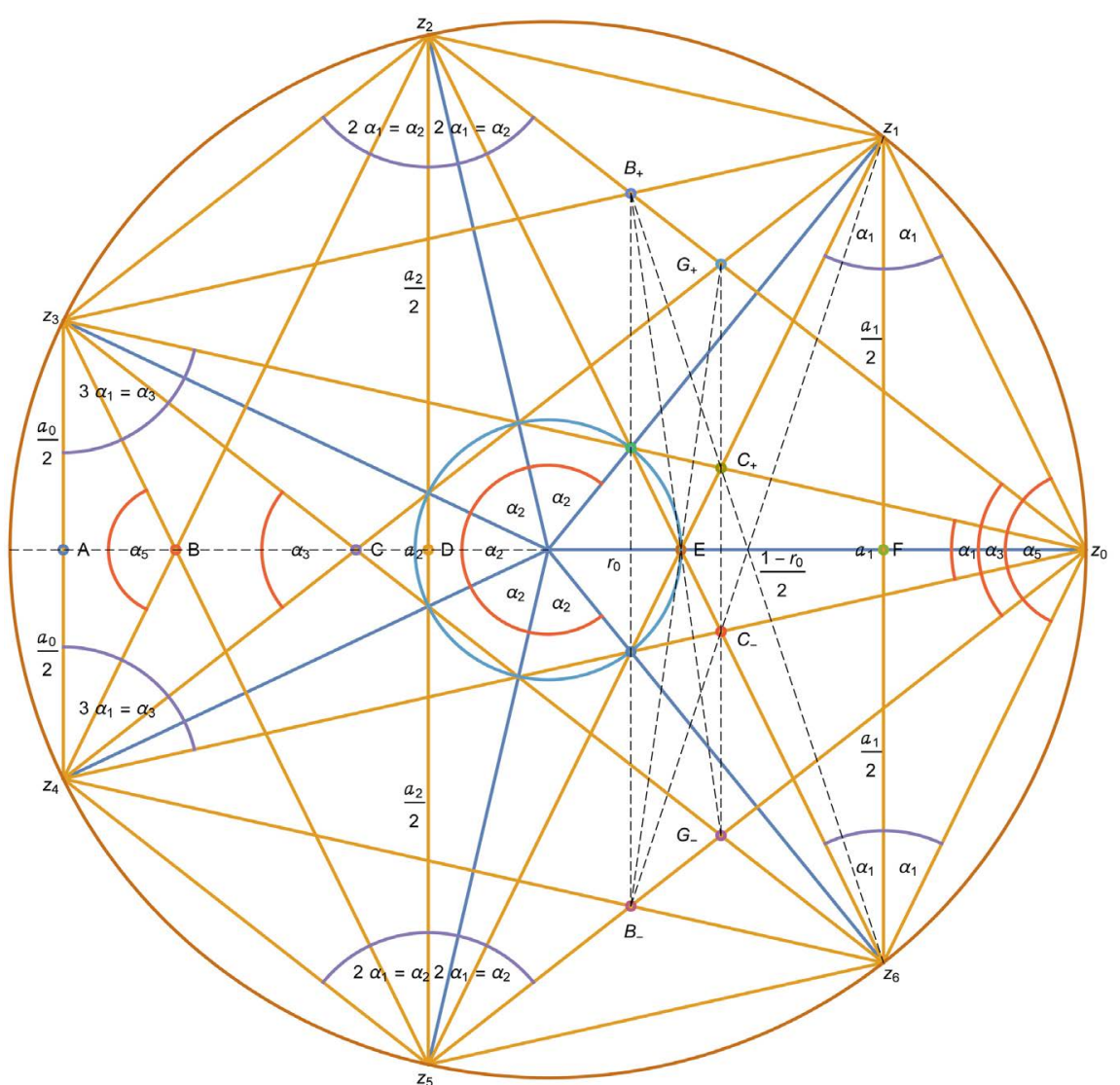

Figure 3. Geometry of regular heptagon. The radius of the circumscribed circle is set $R=1$. The notation of the angles is $\alpha_{m}=m \alpha_{1}=m \frac{\pi}{7}$ and thus $\alpha_{1}=\frac{\pi}{7}$ and, for example, $3 \alpha_{2}=2 \alpha_{3}=6 \alpha_{1}$. Furthermore, $z_{0}=z_{7}=1=R$. The points $B_{ \pm}$are equivalent to $B$ by clock-wise rotation with angle $\pm \frac{4 \pi}{7}$ around the center and $C_{ \pm}$are equivalent to $C$ by clock-wise rotation with angle $\frac{6 \pi}{7}$ around the center. The relations between the angles are easily to verify using Figure 1 . The details of the notation of the points are explained in (6.7), (6.8) and (6.9). 
The points $B_{ \pm}, C_{ \pm}$which are rotated points $B, C$ are at

$$
\begin{gathered}
B_{ \pm}=(0.153989 \pm \mathrm{i} 0.674671), \\
C_{ \pm}=(0.321552, \pm \mathrm{i} 0.154851),
\end{gathered}
$$

and the points $G_{ \pm}$which are the intersection of chords between $Z_{0}=1$ and $Z_{ \pm 2}$ and between $Z_{ \pm 1}$ and $Z_{ \pm 4}$ (i.e., between corner points of the regular heptagon such as given plus equivalent ones by rotations about multiple angles of $\left.\frac{2 \pi}{7}\right)$

$$
G_{ \pm}=(0.321552, \pm 0.541044) .
$$

It is sometimes not easy to find the simplest form of complicated trigonometric expressions due to the many identities where "simplest" in addition is not exactly defined.

We now consider a first "ring" or "generation" of 7 regular heptagons which coincide at one side with the heptagon of equal side-length in the center (see Figure 4). First, it is favorable to establish the centers of the regular pentagons around the coordinate origin. For example, the two centers of the hexagon to the right are at $2 \cos \left(\frac{\pi}{7}\right) \exp \left( \pm i \frac{\pi}{7}\right)$ and the remaining centers are found by rotations from them. The seven regular heptagons at the first ring possess a small overlap which later in parqueting of the whole plane play a role and have to be removed. The points in Figure 4 are determined by

$$
\begin{aligned}
A & =\left(-1-2 \cos \left(\frac{\pi}{7}\right), 0\right)=(-2.80194,0) \\
B & =\left(\left(1+2 \cos \left(\frac{\pi}{7}\right)\right) \cos \left(\frac{5 \pi}{7}\right), 0\right)=(-1.74698,0), \\
C & =\left(-\cos \left(\frac{\pi}{7}\right), 0\right)=(-0.900969 .0) \\
D & =\left(\cos \left(\frac{4 \pi}{7}\right), 0\right)=(-0.222521,0), \\
E & =\left(\left(1+2 \cos \left(\frac{\pi}{7}\right)\right) \cos \left(\frac{3 \pi}{7}\right), 0\right)=(0.62349,0), \\
F & =\left(2 \cos { }^{2}\left(\frac{\pi}{7}\right)+\cos \left(\frac{3 \pi}{7}\right), 0\right)=(1.84601,0), \\
H & =\left(\left(1+2 \cos \left(\frac{\pi}{7}\right)\right) \cos \left(\frac{\pi}{7}\right), 0\right)=(2.52446,0) \\
G & =\left(-\cos \left(\frac{\pi}{7}\right)+\frac{\sin \left(\frac{\pi}{7}\right)+2 \sin \left(\frac{3 \pi}{7}\right)}{\operatorname{tg}\left(\frac{3 \pi}{14}\right)}, 0\right)=(2.08815,0) \\
& ((1)
\end{aligned}
$$




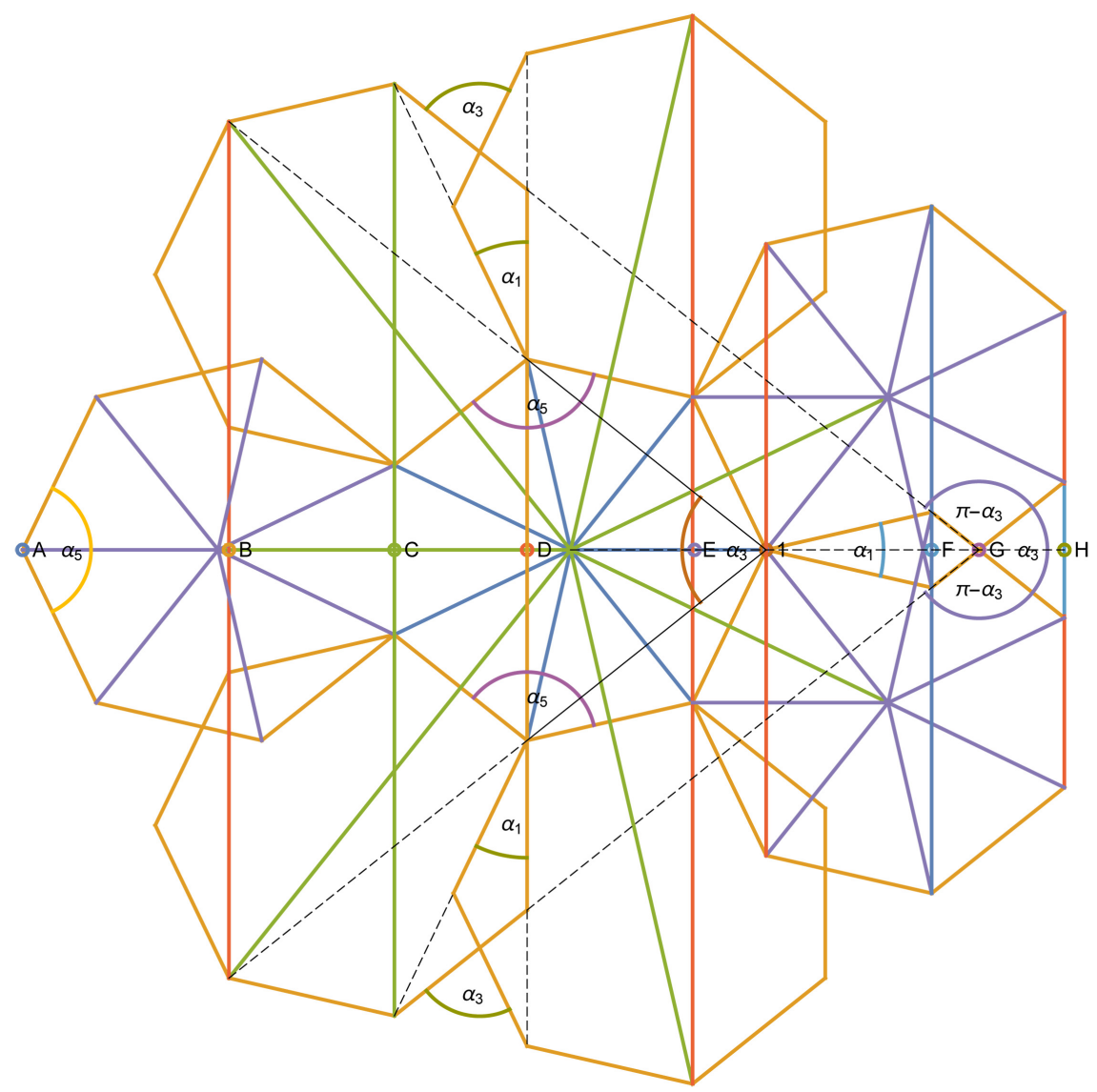

Figure 4. Geometric relations for the first ring of neighbored regular heptagons with overlaps. The angles $\alpha_{m}$ are defined as in Figure 3 that means by $\alpha_{m}=m \alpha_{1}=\frac{m \pi}{7}$. Other geometrical details are explained in (6.10).

If we look to Figure 3 and to Figure 4 we see that when drawing all interesting lines between corner points we have astonishingly many cases of intersection of more than 2 lines in one point.

\section{Two Regular Star-Heptagons and a Semi-Regular Star-Heptagon}

Besides the regular heptagon there are possible two regular star-heptagons shown in Figure 5. They can be obtained if we first draw the 7 corner points of a regular heptagon and if we then connect by lines each corner point with the next $\{7 / 1\} \equiv\{7\} \quad$ (regular heptagon), with the over-next (first star-heptagon $\{7 / 2\}$ ) or with the third-next corner point (second star-heptagon $\{7 / 3\}$ ) and prolong them up to intersection of the lines (the symbols for the star-heptagons are the Schläfli symbols). If we circumscribe around them circles with radius $R=1$ then the side length of these star-heptagons are solutions $\left(u_{3}=2 y_{3}, u_{2}=2 y_{2}, u_{3}=2 y_{3}\right)$ of (4.7) to $(\{7\},\{7 / 2\},\{7 / 3\})$. In the drawing we show here their inner and outer angles. With radius $r=1$ of the inner drawn circles the distances from the center to the tip of the jags $D_{1}$ and $D_{2}$ are, respectively 

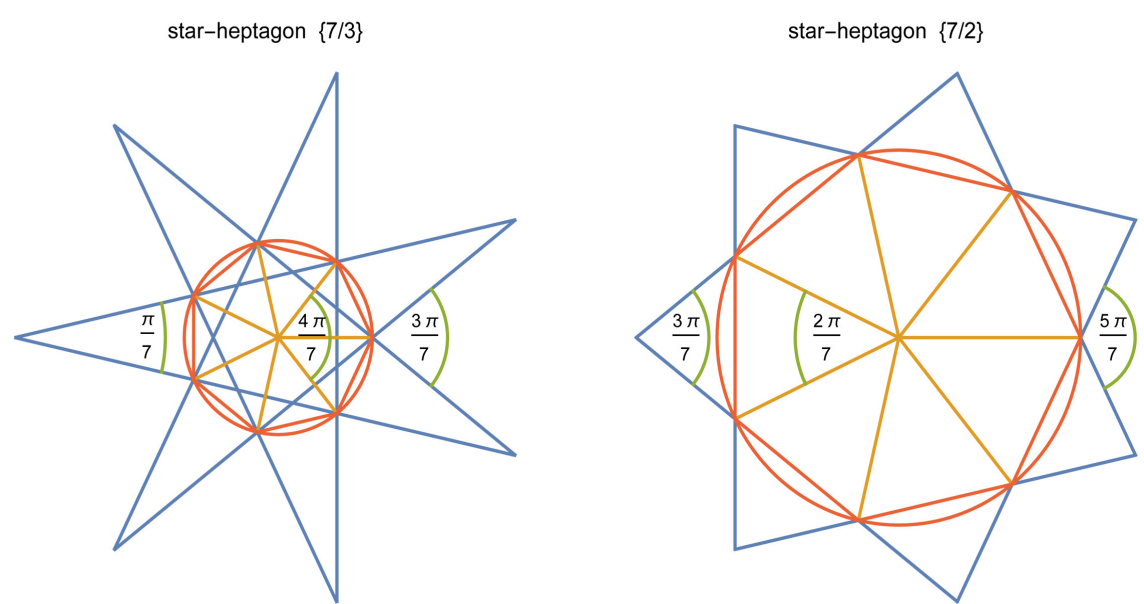

Figure 5. The two regular star-heptagons. For the first star-heptagon the inner angle of a jag is $\alpha_{1}=\frac{\pi}{7}$ and the angle between two jags $\alpha_{3}=\frac{3 \pi}{7}$. For the second star-heptagon the inner angle of a jag is $\alpha_{3}=\frac{3 \pi}{7}$ and the angle between two jags $\alpha_{5}=\frac{5 \pi}{7}$. If the radius of the circle around the inner regular heptagon is set $R=1$ then the tip of the jags possess the distance from the center for the first $D_{1}=1+2 \cos \left(\frac{\pi}{7}\right)=2.80194$ and for the second $D_{2}=1+2 \sin \left(\frac{\pi}{14}\right)=1.44504$.

$$
\begin{aligned}
& D_{1}=\cos \left(\frac{\pi}{7}\right)+\operatorname{ctg}\left(\frac{\pi}{14}\right) \sin \left(\frac{\pi}{7}\right)=1+2 \cos \left(\frac{\pi}{7}\right)=2.80194 \\
& D_{2}=\cos \left(\frac{\pi}{7}\right)+\operatorname{ctg}\left(\frac{3 \pi}{14}\right) \sin \left(\frac{\pi}{7}\right)=1+2 \sin \left(\frac{\pi}{14}\right)=1.44504
\end{aligned}
$$

If we look to Figure 5 we find the following possible calculation. An inner sector of the regular heptagon and the corresponding outer jag possess the common basis which is the side length of the regular heptagon and is $2 \sin \left(\frac{\pi}{7}\right)$ with the perpendicular bisector $\cos \left(\frac{\pi}{2}\right)$. The perpendicular bisector of the jags to the common side length with the regular heptagon is the Cotangent of the half angle of the jag, i.e. $\operatorname{ctg}\left(\frac{\pi}{14}\right)$ or $\operatorname{ctg}\left(\frac{3 \pi}{14}\right)$, respectively, multiplied with the half of the side length of the regular heptagon that means with $\sin \left(\frac{\pi}{2}\right)$. The sum of both mentioned expression is the distance of the tip of the jags to the center.

If we draw around the inner regular heptagon or around the inner star-heptagon $\{7 / 2\}$ a "densely" packed ring of regular heptagons (first generation) that is only possible with small overlaps of the heptagons then we arrive at the two upper pictures in Figure 6. After resection of the overlaps we obtain the two lower pictures in Figure 6. We consider this as two dual cases to each other 

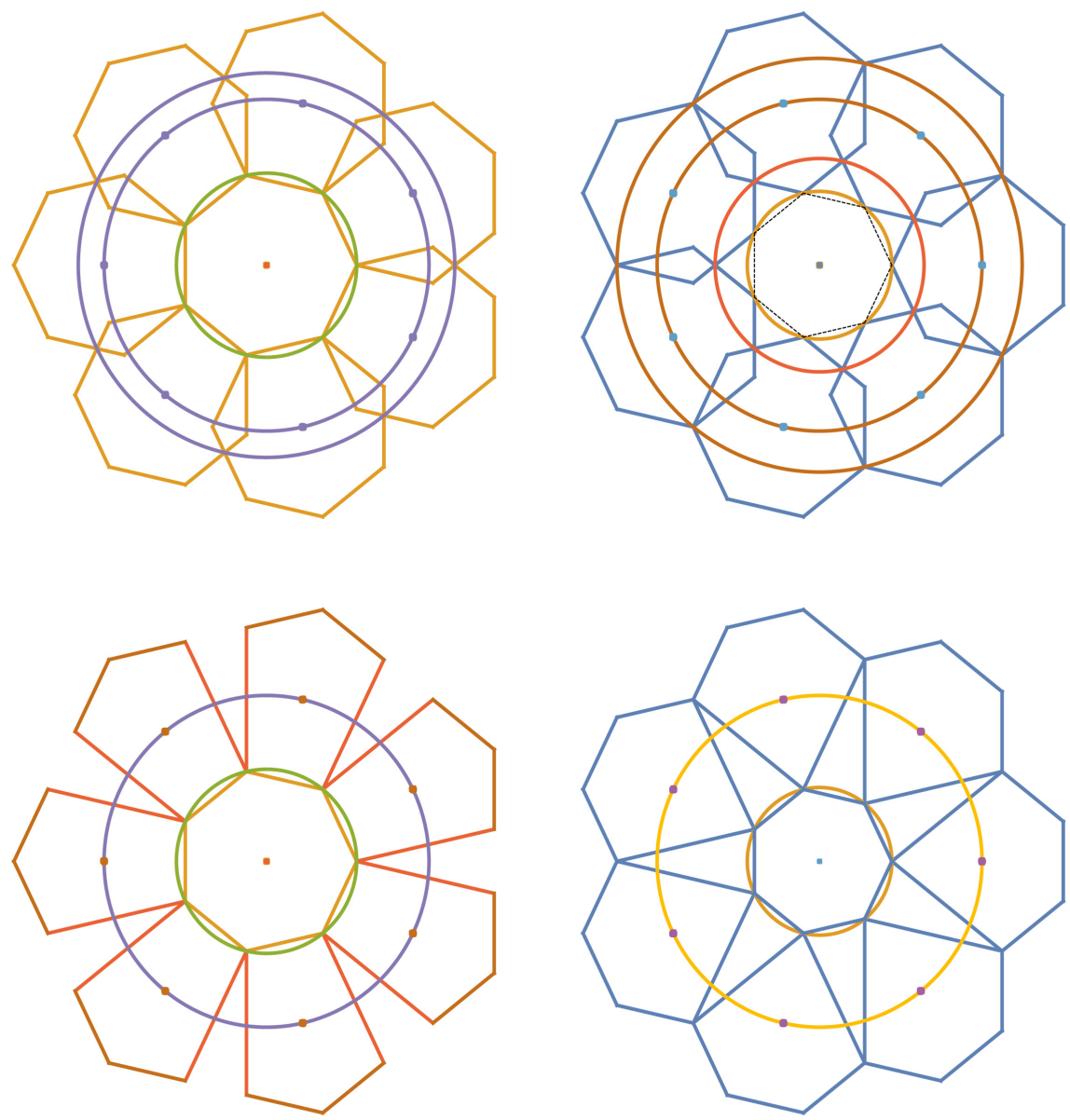

Figure 6. Basic rings of the two alternative parquets. The 7 regular heptagons ring around a central regular heptagon or a star-heptagon, respectively, are densily arranged that is only possible with overlaps as shown in the upper row. These overlaps can be cut in a way, for example, as shown in the lower row. The angles at the outer concave points of the figures are $\frac{3 \pi}{7}, \frac{5 \pi}{7}, \frac{\pi}{7}, \frac{5 \pi}{7}$ (from left to right) which are the same as the inner angles of the jags and the outer angles between two neighbored jags as seen in Figure 5. If the radius of the circles around the regular heptagons is set equal to $R=1$ in the figure on the left-hand side then the distance to the marked centers of the regular heptagons on the first ring is $D=2 \cos \left(\frac{\pi}{7}\right)=1.8019377$ in all partial figures.

that later leads to two dual forms of possible parqueting. In relation to the inner regular heptagons the rings of heptagons in the first generation have different positions in the two cases, in first case they have a common side and in second case the tips of the heptagon of the ring touch the middles of the sides of the central heptagon. If the regular heptagons of the first generation possess the same size in both cases then their distance of their centers from the coordinate origin is the same (see Figure 6).

There is yet another principal way of finding a structure element for patterns with sevenfold symmetry. For this purpose we made Figure 7. We draw here circles around the regular heptagons and lines from their corners to their centers 

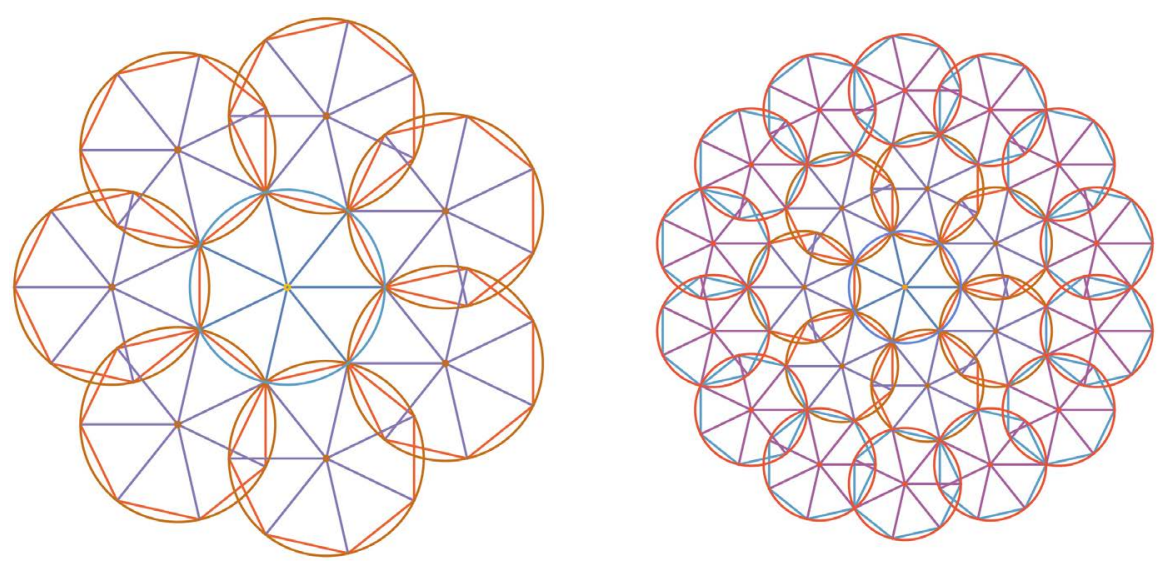

Figure 7. Basic constructions with densely packed regular heptagons and circles around them. In the center of the left-hand figure we recognize a semi-regular star-heptagon which is separately shown in Figure 8 on the left-hand side. We have here small overlaps of the circles. This is also the basis of the parqueting of the whole plane dealt with in Section 8 .

and show the rings of the first and second generation of regular heptagons. It is seen that by parts of the lines appear patterns in form of a star-heptagon which does not belong to the regular star-heptagons. We call it semi-regular star-heptagon. It is shown in Figure 8 on the left-hand side. On the right-hand side of Figure 8 it is shown a ring of the first generation of regular heptagons which touch with their tips the tips of the semi-regular heptagon in the center. It can serve as starting point for further rings with generations of heptagons which together with triangles may cover the whole plane with patterns of sevenfold symmetry. However, the last is not really made up to now.

The geometry of the semi-regular star-heptagon is to see in Figure 8. Two length characteristics from which can be easily calculated other length characteristics are the radius of the circumscribed circle around the regular heptagon in the center which we set $R=1$ and the distance $D$ from the center to the tip of the jags of the semi-regular star-heptagon which is then

$$
D=2 \cos \left(\frac{\pi}{7}\right)=1+\frac{\cos \left(\frac{3 \pi}{14}\right)}{\cos \left(\frac{\pi}{14}\right)}=1.80194 \text {. }
$$

The inner angles of the jags of the semi-regular star-heptagon are $\frac{2 \pi}{7}$ and the angles between their jags $\frac{4 \pi}{7}$ and they fit together insofar as they are in a relation 1:2. However, it is not possible to cover the whole plane only with semi-regular star-heptagons. From Figure 9 it can be seen that this is only possible with overlaps and (or) gaps between these star-heptagons. The outer 14 angles at the concave points on the border in right-hand picture are $\frac{6 \pi}{7}$ and 

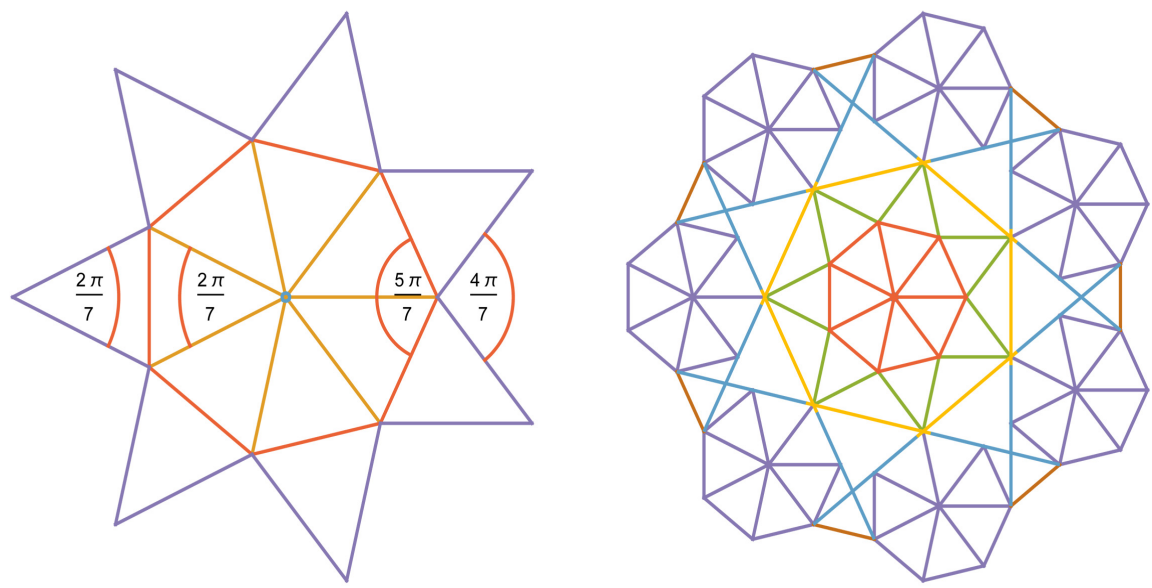

Figure 8. The semi-regular star-heptagon and a ring of regular heptagons around it without overlaps. In the center of the right-hand figure we recognize a semi-regular star-heptagon which is separately shown in the figure on the left-hand side. If the radius of the circumscribed circle around the inner regular heptagon is set $R=1$ then the tip of the jags in the figure of the left-hand side possess a distance to the center of $D=2 \cos \left(\frac{\pi}{7}\right)=1.80194$ and in the right-hand picture from the center to the centers of the ring of regular heptagons of $D_{2}=1+2 \cos \left(\frac{\pi}{7}\right)=2.80194$. The outer angles at the 14 (concave) corner points are $\frac{6 \pi}{7}$. Therefore the inner angles of the jags, the angle between the jags and the outer angles at the concave corner points of the border in the figure on the right-hand side possess a relation 1:2:3. The border line between two neighbored regular heptagons is exactly equal to the side length of the regular heptagon that we prove in the text.
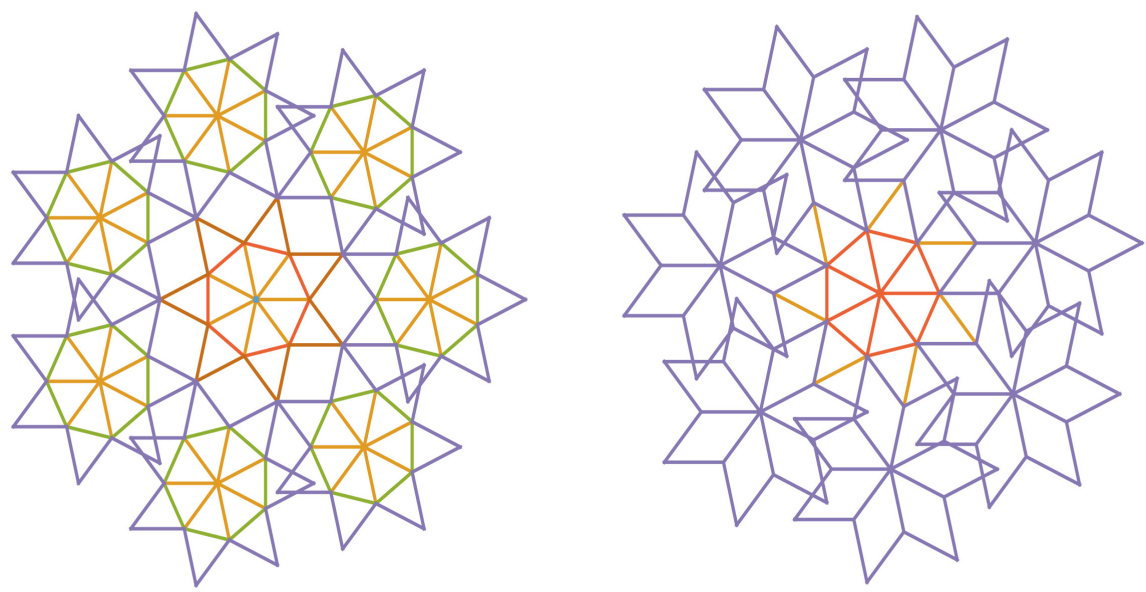

Figure 9. Basic constructions with densely packed semi-regular heptagons but with overlaps. In the center of the two figures we recognize semi-regular star-heptagons which are separately shown Figure 8. If the radius of the circumscribed circle around the inner regular heptagon is set $R=1$ then the tips of the inner jags possess a distance to the center of $D=2 \cos \left(\frac{\pi}{7}\right)=1.80194$. The left-hand figure possesses symmetry $C_{7_{V}}$ with mirror symmetry and the right-hand figure only the lower symmetry $C_{7}$. Both arrangements possess overlaps. 
together with the inner angles of the jags and the angles between the jags of the semi-regular heptagon are in a relation 1:2:3 that opens possibilities to order further rings of elements with sevenfold symmetry around the first ring without overlaps.

We now prove that the borderline (in brown) between two outer regular heptagons is exactly equal to the side length of the regular heptagon as it seems from right-hand Figure 8. From right-hand side in Figure 8 we see that this length of the borderline is the distance $D\left(z_{+}, z_{-}\right)$of the following two complex points $z_{+}$and $z_{-}$within the right-hand angle sector of $\frac{2 \pi}{7}$.

$$
z_{ \pm}=\left\{1+2 \cos \left(\frac{\pi}{7}\right)\right\} \exp \left( \pm \mathrm{i} \frac{\pi}{7}\right)+\exp \left(\mp \mathrm{i} \frac{2 \pi}{7}\right)
$$

which is

$$
\begin{aligned}
D\left(z_{+}, z_{-}\right) & =2\left\{1+2 \cos \left(\frac{\pi}{7}\right)\right\} \sin \left(\frac{\pi}{7}\right)-2 \sin \left(\frac{2 \pi}{7}\right) \\
& =2 \cos \left(\frac{5 \pi}{14}\right)=2 \sin \left(\frac{\pi}{7}\right)=D\left(z_{1}, z_{0}\right)=0.867767,
\end{aligned}
$$

where $z_{0}$ and $z_{1}$ are roots of the cyclotomic equation (see (3.5)). It is exactly equal to the side length of the regular heptagons with circumscribed circle with radius $R=1$. Therefore, in the next "ring" of polygons in the right-hand Figure 8 we may place 7 new regular heptagons with these borderlines as basis. On the other side it is clear from this Figure 8 that if we place on the neighbored regular heptagons to these borderlines 14 new regular heptagons of the same size then they meet them with their tips at the distance $D=\left\{1+2 \cos \left(\frac{\pi}{7}\right)\right\} 2 \cos \left(\frac{\pi}{7}\right)=5.04892$ from the center.

\section{Parqueting of the Whole Plane with Seven-Fold Symmetry $C_{7 v}$ by Tiles of 4 Different Types}

We consider now ways of parqueting of the whole plane with a center of point-group symmetry $C_{7 v}$. Rings with 7, 14, 21 and so on regular heptagons are arranged in a compact way but with overlaps and gaps that is shown in Figure 10. The parts which have to be removed are shown by red lines in Figure 11 and the gaps in form of small rhombi can be left as small tiles or can be cut into 4 equal parts and added to the remaining tiles. In first form the whole plane will be filled with two sorts of polygons, irregular heptagons and rhombi and in second form by only irregular pentagons in the whole pattern. This is shown in Figure 12. As mentioned we call each new "ring" (better quasi-rings) of new tiles a new generation of tiles and if we call the regular heptagon in the center the 0 -th generation the first generation possesses 7 tiles and each following generation possesses 7 tiles more. Thus the ring of the $k$-th generation is formed by $7 k$ tiles. In Figure 10, Figure 11 are drawn 4 generations of tiles up to $k=4$ and in Figure 12 also 4 generations but from $k=2$ up to $k=5$. 

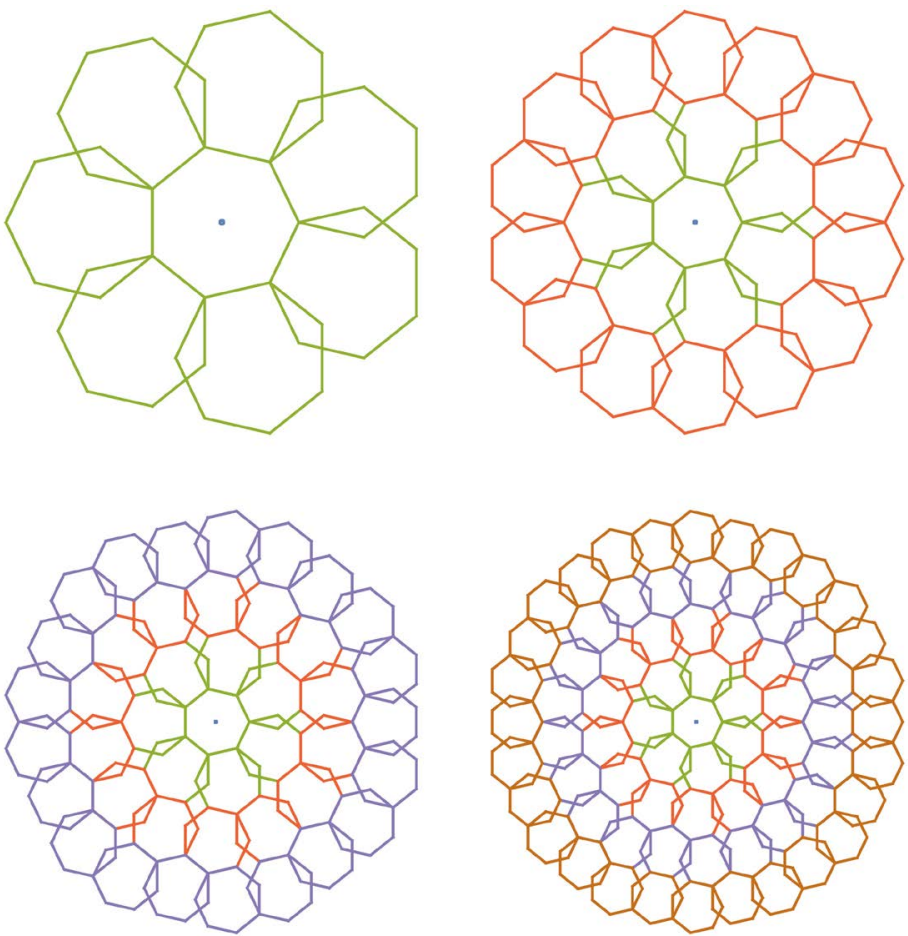

Figure 10. Covering of plane with densely packed regular heptagon tiles. The regular heptagons possess small overlaps from the first generation (rings) on and small gaps between 4 neighbored ones. The point-group symmetry is $C_{7_{V}}$ that mean $C_{7}$ plus 7 additional mirror lines. In the partial figures are drawn up to 4 generations (rings) of regular heptagons.
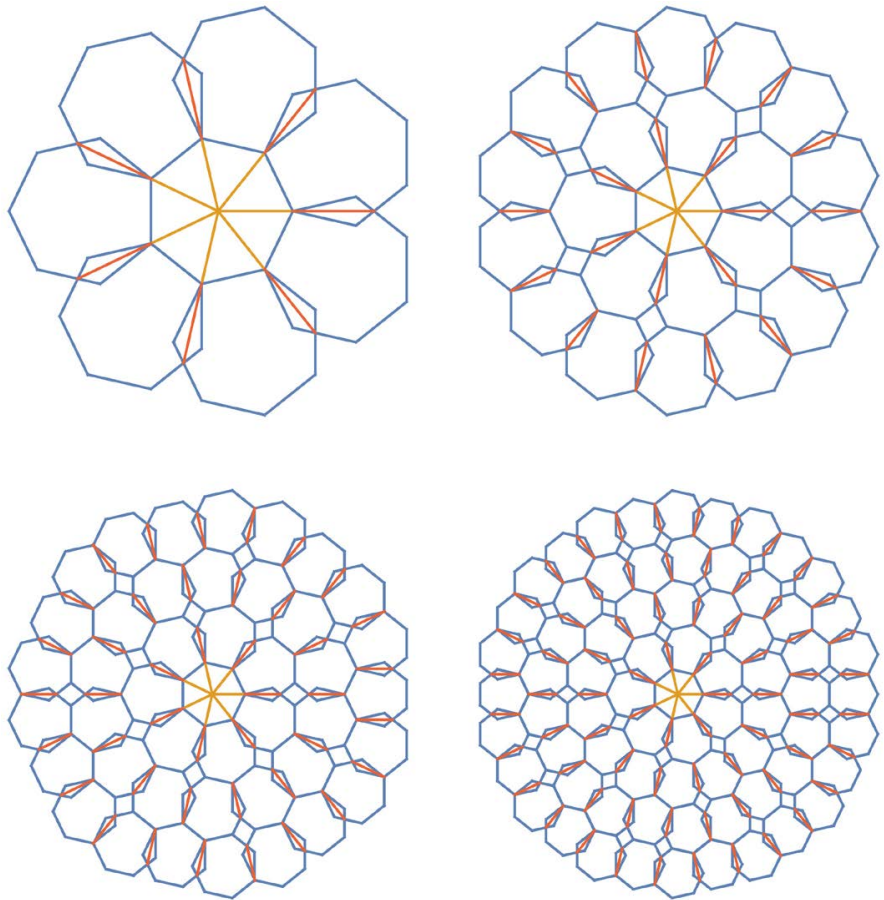

Figure 11. Removing the overlaps and transition to covering of plane with irregular heptagons and rhombuses. If the tiles are cut off along the red lines one gets irregular heptagons with mirror symmetry. 

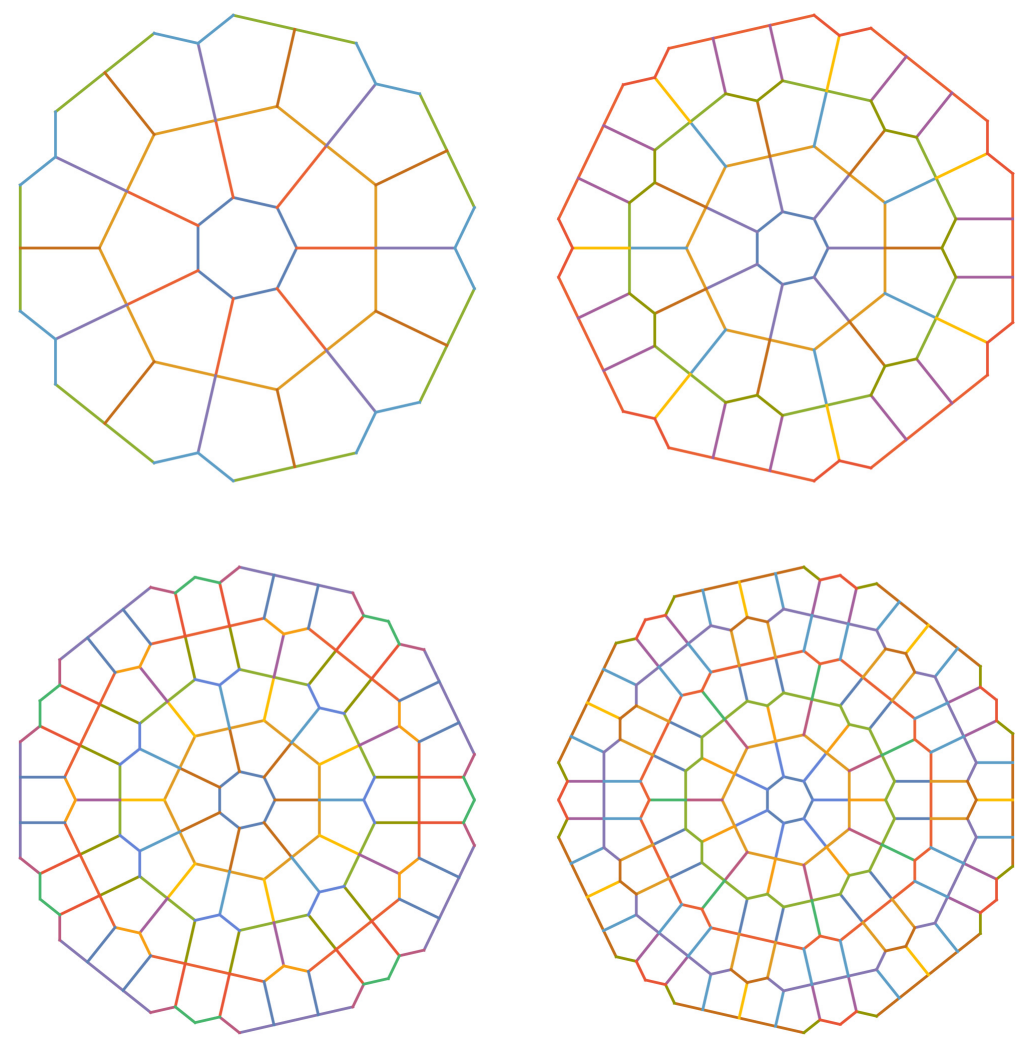

Figure 12. Parqueting of plane with seven-fold symmetry by non-regular pentagons of 4 different forms. Each generation adds $7 n$ new tiles to the preceding generation but the whole figures become more similar with their boundaries to regular heptagons for high $n$. There are shown here 5 generations (rings) of tiles. If the central heptagon belongs to $n=0$ our four partial pictures begin with the first generation that means with $n=2$ and goes up to $n=5$. The total sum $S_{n}$ with tiles of $n$ generations is described by the formula $S_{n}=\frac{7 n^{2}+7 n+2}{2}$ that leads to $S_{n}=\{1,8,22,43,71,106, \cdots\}$. This means 106 tiles up to the 5-th generation in the last partial figure.

The distance of two next neighbored centers of the regular heptagons in Figure 10 in different generations of rings is $D=2 \cos \left(\frac{\pi}{7}\right)$. The direction of their position from the coordinate origin in the complex plane is obtained by multiplication of this distance by complex numbers which are obtained in following way. One begins from coordinate origin and goes to the centers from neighbor to next neighbor and add for each step a factor $\exp \left(\right.$ in $\left.\frac{\pi}{7}\right),(n=0, \pm 1, \pm 2, \cdots, 6)$ where $n \frac{\pi}{7}$ is the angle between the coordinate center to the center of the next considered regular heptagon. Since there are different ways from coordinate origin to the center of the considered regular heptagons over neighbored we have different possibilities of summations which have to lead to the same result. We consider a few first generations and denote the distances from the coordinate center to the centers of the regular heptagons in Figure 12 by $D_{l}^{k}$ where upper index $k$ means the $k$-the generation and lower 
index $l=0, \pm 1, \pm 2, \cdots$ enumerates the different possibilities. The complex centers in the $k$-th generation we denote by $c_{1}^{k}=a_{1}^{k}+\mathrm{i} b_{1}^{k}, c_{2}^{k}=a_{2}^{k}+\mathrm{i} b_{1}^{k}, \cdots$ and the corresponding angles by $\gamma_{l}^{k}$. If we are only interested in the distances and in the angles to the positive $x$-axes we may only consider a sector of angle $\frac{2 \pi}{7}$ and obtain all other cases by rotations about multiples of angle $2 \frac{\pi}{7}$. In this way we find for the first 3 generations:

1) generation

$$
\begin{gathered}
c_{ \pm 1}^{1}=2 \cos \left(\frac{\pi}{7}\right) \exp \left( \pm \mathrm{i} \frac{\pi}{7}\right), \\
D_{ \pm 1}^{1}=2 \cos \left(\frac{\pi}{7}\right)=1.80194, \quad \operatorname{tg}\left(\gamma_{ \pm 1}^{1}\right)= \pm \frac{\sin \left(\frac{\pi}{7}\right)}{\cos \left(\frac{\pi}{7}\right)},
\end{gathered}
$$

2) generation

$$
\begin{gathered}
c_{ \pm 1}^{2}=2 \cos \left(\frac{\pi}{7}\right)\left\{1+\exp \left( \pm \mathrm{i} \frac{\pi}{7}\right)\right\}, \\
D_{ \pm 1}^{2}=2 \cos \left(\frac{\pi}{7}\right) 2 \cos \left(\frac{\pi}{14}\right)=3.51352, \quad \operatorname{tg}\left(\gamma_{ \pm 1}^{2}\right)= \pm \frac{\sin \left(\frac{\pi}{7}\right)}{1+\cos \left(\frac{\pi}{7}\right)},
\end{gathered}
$$

3) generation

$$
\begin{gathered}
c_{0}^{3}=2 \cos \left(\frac{\pi}{7}\right)\left\{\exp \left(-\mathrm{i} \frac{\pi}{7}\right)+1+\exp \left(\mathrm{i} \frac{\pi}{7}\right)\right\}, \\
D_{0}^{3}=2 \cos \left(\frac{\pi}{7}\right)\left(1+2 \cos \left(\frac{\pi}{7}\right)\right)=5.04892, \operatorname{tg}\left(\gamma_{0}^{3}\right)=0, \\
c_{ \pm 1}^{3}=2 \cos \left(\frac{\pi}{7}\right)\left\{1+2 \exp \left( \pm \mathrm{i} \frac{\pi}{7}\right)\right\}, \\
D_{ \pm 1}^{3}=2 \cos \left(\frac{\pi}{7}\right) \sqrt{5+4 \cos \left(\frac{\pi}{7}\right)}=5.28551, \quad \operatorname{tg}\left(\gamma_{ \pm 1}^{3}\right)= \pm \frac{2 \sin \left(\frac{\pi}{7}\right)}{1+2 \cos \left(\frac{\pi}{7}\right)} .
\end{gathered}
$$

All shown pictures of parqueting possess the point-group symmetry $C_{7 \mathrm{~V}}$ (7-fold rotation around the symmetry center plus mirror symmetry to 7 lines through the center, i.e. 14 symmetry elements). If we do not remove the small rhombic gaps which are seen in Figure 11 but only the overlaps then we get a parqueting without destroying the symmetry $C_{7 V}$ but with irregular heptagon plus rhombic tiles tiles of the form of the gaps. One may lower this symmetry to $C_{7}$ if we remove the mirror symmetries by making, e.g., bulges at the tiles in a way that after this they fit together and do not form gaps in the parquet. There is a lot of possibilities for changing this parqueting hardly to overview. 


\section{Possible Tile Forms for Parqueting with Seven-Fold Symmetry}

We now discuss possible forms of the tiles for parqueting the whole plane with seven-fold point-group symmetry $C_{7 v}$ with full covering.

Apart from the regular heptagon in the center we have only 4 other types of tiles in form of not fully regular pentagons for covering the whole plane by a parquet. These 4 tile forms are shown in Figure 13. The first two tiles, we call them "Lantern" and "Häusle", possess a mirror line as symmetry. The second two tiles, we call them "Kite 1" and "Kite 2" are two mutually enantiomorphic forms of an asymmetric pentagon. The 4 forms of tiles are best understood if we show them in connection with the regular heptagon (red color) from which they are made by changes of their form. Interesting is that all 4 types of tiles possess 2 right angles which are signified by double rings in Figure 13.
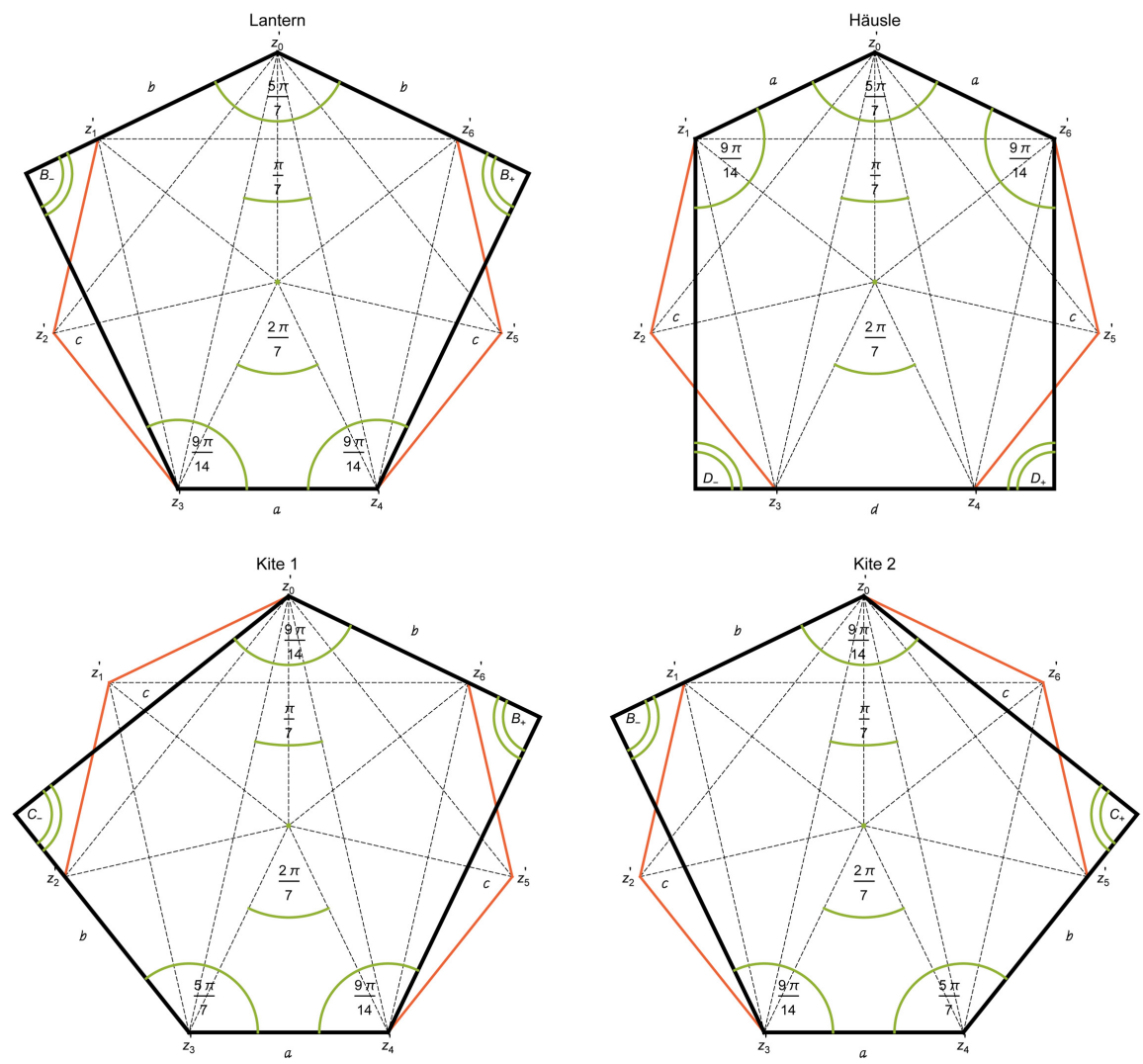

Figure 13. The four basic pentagon tiles for a parquet with seven-fold point-group symmetry. Each tile contains two right angles, two angles $\frac{9 \pi}{14}$ and one angle $\frac{5 \pi}{7}$. The two "Kites" are enantiomorphic forms meaning that they are mutually mirror-symmetric. The angle sum $S_{N=5}$ (sum of inner angles at the corners) of an arbitrary pentagon is equal to $3 \pi$ and, generally, for an arbitrary $N$-gon equal to $S_{N}=(N-2) \pi$. The "Häusle" contains two sides of the regular heptagon with side-length $a$ the others contain exactly one side but some other sides are along some sides of the regular heptagon. By slicing the pentagons along the dashed lines one obtains more simple basic building stones for tessellation. 
Furthermore, all types of tiles have at least parts of the basic regular heptagon in common.

If the radius of the heptagon is equal to 1 as before than the side length of the heptagon is $a=2 \sin \left(\frac{\pi}{7}\right)$ and the tiles possess only two different side-length which we denote by $b$ and $c$. Their lengths are

$$
\begin{aligned}
& a=2 \sin \left(\frac{\pi}{7}\right)=0.867767, \\
& b=2 \cos \left(\frac{\pi}{14}\right) \sin \left(\frac{3 \pi}{14}\right)=\sin \left(\frac{2 \pi}{7}\right)+\sin \left(\frac{\pi}{7}\right)=1.215715, \\
& c=2 \cos \left(\frac{\pi}{14}\right) \cos \left(\frac{3 \pi}{14}\right)=\cos \left(\frac{2 \pi}{7}\right)+\cos \left(\frac{\pi}{7}\right)=1.524459 .
\end{aligned}
$$

The angles within the corners of the tiles in form of pentagons are shown in Figure 13. It is interesting that each tile form possesses two right angles signified by double circles.

One may modify the tiles covering the plane without leaving gaps but preserving the symmetry $C_{7}$ or at least $C_{v} \equiv C_{s}$ in many ways. The center can be modified by substitution of the regular heptagon by the two possible star-heptagons. Then we have also to change the polygons of the first generation but the further generations may remain the same ones. Removing some separation lines in the higher generations one may obtain the larger tiles of different forms. Another possibility is to divide the tiles into two equal parts and color one parts black and the other part red. In this way one obtains the simplest extension of the symmetry $C_{2 v}$ to a color symmetry.

We now give the numbers of the different types of tiles which are needed in each generation. This is given by Table 1 .

For high order of generations the number of tiles of the "Häusle"-type becomes predominant and the border of parquet becomes more and more similar to a regular heptagon with small damages at the edges and from generation to next higher generation the position of the border rotates by an angle of $\frac{\pi}{7}$. For the total number of tiles $S_{n}$ up to the $n$-th generation follows then

Table 1. Numbers of dierent tiles in parquet.

\begin{tabular}{ccccc}
\hline Ring Nb. & Lantern & Kite $1+2$ & Häusle & Sum \\
\hline$k=1$ & 7 & 0 & 0 & 7 \\
$k=2$ & 0 & $7+7$ & 0 & 14 \\
$k=3$ & 0 & $7+7$ & 7 & 21 \\
$k=4$ & 0 & $7+7$ & 14 & 35 \\
$k=5$ & 0 & $7+7$ & 21 & 42 \\
$k=6$ & 0 & $7+7$ & 28 & $\vdots$ \\
$\vdots$ & $\vdots$ & $7+7$ & $\vdots$ & $7 k$ \\
\hline
\end{tabular}




$$
S_{n}=1+\sum_{k=1}^{n} 7 k=1+\frac{7 n(n+1)}{2}=\{1,8,22,43,71,106,148,197, \cdots\} .
$$

This arithmetic sequence $S_{n}$ contains prime numbers as well as composite numbers.

It is clear that the tiles can be varied in an infinite number of ways, for example, by omission of separating lines or addition of new separating lines in symmetric way (deflation and inflation [30]).

\section{A Second Dual Form of Parqueting with Seven-Fold Symmetry}

There exists a second basic covering of the plane with seven-fold symmetry $C_{7 V}$ which in certain sense is dual to the covering considered in Figure 12. The starting point from a regular star heptagon $\{7 / 2\}$ in the center (or also $\{7 / 3\}$; see Figure 6) is shown with overlapping by the arrangement of regular heptagons in Figure 14. The overlaps and the gaps can be removed in similar way as this was made from Figures 10-12 in the formerly considered case. We do not draw here these next steps since they are clear. The distance from the symmetry center
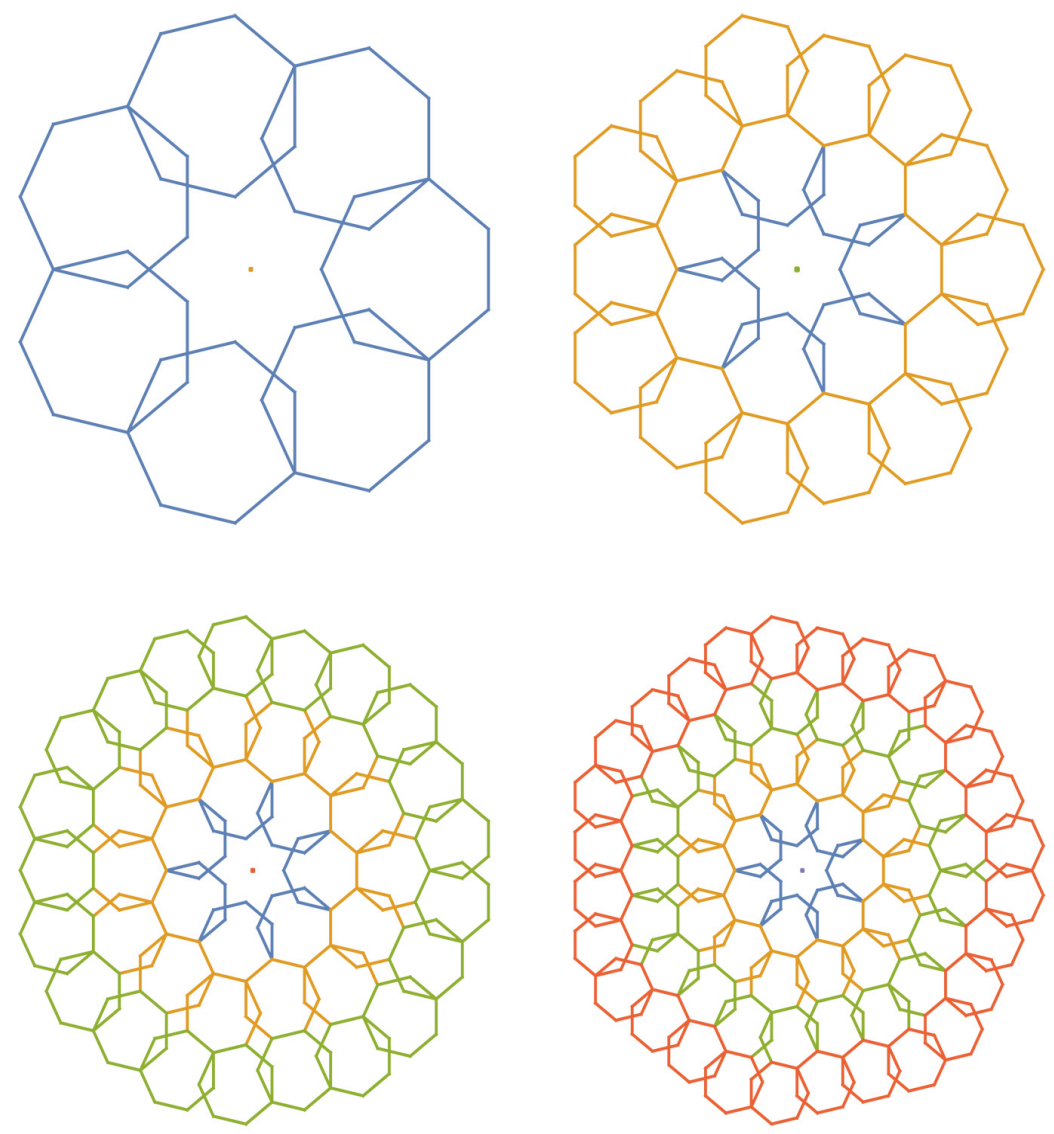

Figure 14. To parqueting of whole plane in seven-fold symmetry in alternative form. One may consider this parqueting in some sense as dual to the parqueting in Figure 12 with the prestep in Figure 10. Due to analogues we did not produce here the corresponding pictures in analogy to Figure 11 and Figure 12. 
(coordinate origin) to the first "ring" of regular heptagons is $D=2 \cos \left(\frac{\pi}{7}\right)$ if the circumscribed radius of the regular heptagons is taken $R=1$ that is the same as in first case but the position of the regular heptagons is now rotated by an angle $\pi$ (or equivalently $\frac{(2 n+1) \pi}{7}$ ).

For the first two generations of tiles depending on cut-off two further tiles in form of non-regular pentagons are possible which are represented on Figure 15. We call them "Kite" and "Birdhouse". These tiles can be also seen in Figure 6 in the lower part. The number of tiles in form of non-regular pentagons after cut-off of the overlaps is analogous to the numbers given in Table 1 .

It is often fascinating for scientists if objects with an infinite number of elements (here translations of elementary cells) can be combined with other elements (here uncountable number of point-group symmetries) only to a finite number of types in such way that the number of types can be listed.

\section{Anti-Symmetry, Shubnikov Groups, Time Inversion and Magnetic and Non-Magnetic Groups}

In this Section we explain shortly anti-symmetry first considered by Heesch and later by Shubnikov and Koptsik and others. It possesses physical importance if the element of anti-symmetry is time inversion which changes the direction of electric currents and the direction (or rotational sense) of the magnetic field and magnetic moments. It preserves the electric charges and the direction of the electric field.

In pure geometry, both considered forms of parqueting with sevenfold symmetry can be extended to antisymmetry or "black-white" symmetry ("white" is sometimes unfavorable for the paper color and often "red" is taken instead for
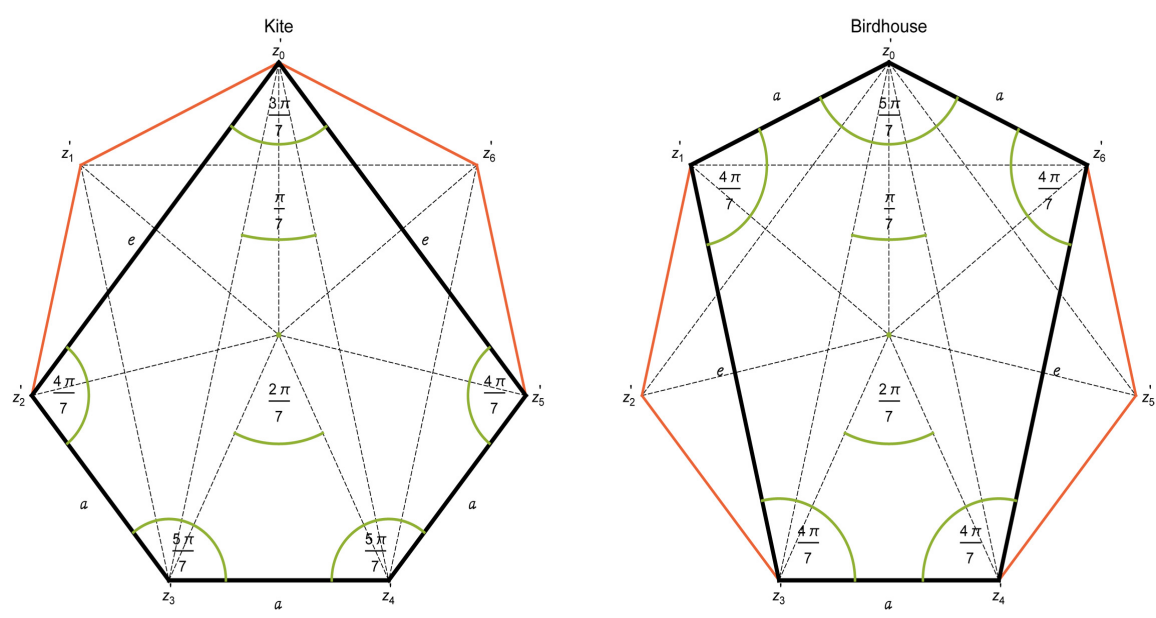

Figure 15. Two additional basic pentagon tiles for a parquet with seven-fold point-group symmetry. Both figures contain three sides of the basic regular heptagon. The angles (no right angle) are shown in the figures. One may recognize these tiles also in the lower pictures of Figure 6. 
graphical representations, e.g., [15]). We mentioned that the group $C_{7 v}$ can be obtained from $C_{14}$ represented in the form

$$
C_{14}=C_{7}+\left(C_{14}-C_{7}\right) \text {, }
$$

by multiplication of the coset $\left(C_{14}-C_{7}\right)$ to the subgroup $C_{7}$ with an element of mirror symmetry $\sigma \equiv m_{v} \in C_{s}$ that means here mirror symmetry with respect to a line through coordinate origin in the plane ( $\sigma$ is the notation of Landau and Lifshits [18] for such an element). This leads to the group structure of $C_{7 V}$

$$
C_{7 v}=C_{7}+\sigma\left(C_{14}-C_{7}\right) \text {. }
$$

with 14 elements. If we substitute herein the group element $\sigma$ by another (independent) element, for example, by the color transition from "black" to "white" then we obtain an antisymmetry group or, equivalently, a color group. Similar to this procedure was the construction in three-dimensional case of the $90=32+$ 58 generalized magnetic and 32 non-magnetic crystal classes where the element of antisymmetry is then the time inversion [17]. In our paper [24] we gave a structure which involves spatial inversion, time inversion and product of spatial and time inversion on an equal level and shows the analogies between magnetic and gyrotropic classes (for optics). In abstract sense of the mathematical group structure the groups (11.1) and (11.2) are isomorph and therefore possess the same number of elements, the same irreducible representations and the same character tables.

The non-magnetic classes are the direct products of a usual class (in our case $C_{7}$ ) with the group of time inversion (two elements: identity element and time inversion; we denote it here $\underline{C}_{i}$ )

$$
\underline{C_{7}}=C_{7} \times \underline{C_{i}},
$$

and possesses 14 elements. This is a non-magnetic class but not a crystal class ${ }^{3}$ (since $C_{7}$ is not compatible with translations) or in case that $C_{i}$ means a group of black-to-white transitions a colorless group. A simple realization is, for example a regular heptagon where each pattern in the basic sectors of angle $\frac{2 \pi}{7}$ is colored with black and red color at once. There are 32 non-magnetic crystal classes and together with the 90 magnetic crystal classes 122 generalized crystal classes. The groups of symmetry and anti-symmetry are called Shubnikov groups. Three basic articles of Shubnikov from 1961-1966 to this topics are republished in [14] (last three articles under "Symmetry", pp. 161-204). Shubnikov used the name "black-white" groups for groups of symmetry and anti-symmetry. This together with rich other material to symmetry is represented in the beautiful monograph of Shubnikov and Koptsik [15]. The voluminous graphical representation of the symmetry elements of all crystallographic space groups of symmetry and anti-symmetry by Koptsik [16] although needed only for very few specialists is a highly intellectual achievement.

By analogous constructions one may obtain color groups with more than two ${ }^{3}$ Whether or not it is somewhere realized in nature is another problem. 
colors. In our case of sevenfold symmetry one may start for $n$ colors from the pure rotation group $C_{7 n}$ and may consider the subgroup $C_{7}$ with all its $n-1$ cosets to this subgroup and may multiply all these $n$ sets by the transition to the $n$ colors in all possible combinations. The color groups are also called Byelov groups in Russian literature.

\section{Periodic Tilings of the Whole Plane, Translations and Net or Lattice Symmetry}

Adding discrete translation symmetry to an elementary cell with point-group symmetry one obtains a lattice, in our case a two-dimensional lattice. Two principal cases are possible, first a group with translations in only one direction (bordures) and second a group with two basic translations in two independent directions (net). It is well known that sevenfold point-group symmetry $C_{7}$ and $C_{7 v}$ combined with discrete translation symmetry is not possible. This is true as well for two-dimensional lattices as also for three-dimensional lattices. Therefore, we may only ask wether or not subgroups of $C_{7}$ and $C_{7 V}$ can be combined with translation symmetry. The point group $G_{7}$ possesses only the genuine subgroup $C_{1}$ (only identity element) and, clearly, it fits to be combined with translation symmetry. The point group $C_{7 \mathrm{~V}}$ possesses as genuine subgroups $C_{7}$ which we already excluded and the subgroup $C_{v} \equiv C_{s}$ which is possible to be combined with translation symmetry. One may choose a part of the picture with point-group symmetry $C_{7 V}$ or $C_{7}$ which at least possesses the group $C_{1}$ (no symmetry) or the mirror symmetry $C_{1 v} \equiv C_{s}$ and declare it as an elementary stripe or an elementary cell and may apply to it a one-dimensional or a two-dimensional translation group.

There exist 17 two-dimensional lattice groups (or reticular ornaments or net groups in Russian literature [16]) which were first derived by E. S. Fyodorov and later by Pólya [12] [20] and correspond to the 230 crystallographic space groups [15] [16] [21]. The 17 net groups are illustrated schematically and (or) by basic patterns, for example, by Steinhaus [10] (chap. 4), Shubnikov and Koptsik [15] (chap. 7, Figs. 149 and 150), Conway et al. [27] (chap. 3, pp. 34-39), Grünbaum and Shephard [26].

The sevenfold point-group symmetry $C_{7 V}$ or $C_{7}$ vanishes if we combine it with translations and the result may possess as maximal point-group the symmetry $C_{s}$ (mirror symmetry) or $C_{1}$ (no symmetry) and it is therefore not simple to obtain aesthetic forms of ornamental bordures or net groups because they do not fit together very well after translation. The chosen elementary stripe or cell may possess arbitrary general form and may degenerate to arbitrary simpler form. Satisfying patterns from an aesthetical point of view for tiling one may obtain by choosing simple elements as, for example, regular heptagons or star-heptagons as content of the elementary cell with some place to the border of the elementary cell and if we then apply to them a translation group.

These remarks may form the transition to the next more important aperiodic 
tilings and to analogues of Penrose tiles but with basic elements of sevenfold symmetry.

\section{Aperiodic Tilings of Whole Plane with Elements of Seven-Fold Symmetry and Penrose Tiling}

Periodic tiling or parqueting of the whole plane with a few types of tiles in form of regular polygons is very restricted in their number. For example, there are only 3 forms with triangle, square and hexagon with which one may cover the whole plane without gaps. All tilings with 2 or more species of regular polygons are also known [10]. For periodic tiling with regular polygons plus some irregular polygons there are more possibilities. In contrast, aperiodic tiling with non-regular polygons admits an infinite number of possibilities which is difficult to overview. It seems to be possible to cover the whole plane with regular heptagons and star-heptagons and additionally other irregular polygons of a low number of species in a way which is analogous to Penrose tiles (e.g., [26] [27] and the first two articles in [30]). A special class of them are asymmetric tilings with a central symmetry. From this type is the tiling or parqueting in Figure 12.

In this Section we consider by a few examples aperiodic tilings of the whole plane with basically elements of sevenfold symmetry that means of regular heptagons and star-heptagons which can be considered as some analogues to Penrose tilings or tessellations. If we look to pictures of aperiodic tessellations by Penrose tiles in the literature, in particular, in [26] [27] [28] and the first two articles in [30], then we see that here basically elements of fivefold symmetry that means that regular pentagons and semi-regular star-pentagons play a main role. Figure 7 shows a possible basis for an analogue with star-heptagons. Clearly, with elements of only fivefold symmetry (regular heptagons and star-heptagons) or seven-fold symmetry alone we cannot cover the whole plane without gaps and there remains a short number of other polygon species which fill the gaps.

Obviously, there are many possibilities to cover the whole plane aperiodically with tiles of basically sevenfold symmetry. We give here an example starting from Figure 16 where seven regular heptagons touch their neighbors in two different ways. Figure 17 shows how we may continue with covering the whole plane by further rings of polygons. However in the left-hand pattern in Figure 17 we have small overlaps which have to be removed. Since it was difficult to see all possibilities in advance we did not calculate the centers of the rings of new generations but experimented by computer with elements such as shown in Figure 18 where we could change some parameters. Figure 8 shows that the semi-regular heptagon with the right-hand figure can be also taken as starting point for aperiodic tilings with basically elements of sevenfold symmetry but this must be investigated in future. The possibilities of aperiodic tiling with basic elements of sevenfold symmetry are by far not exhausted and this can be only the beginning for a more systematic search.

Last but not least let us show for comparison in Figure 19 an example of tiling 

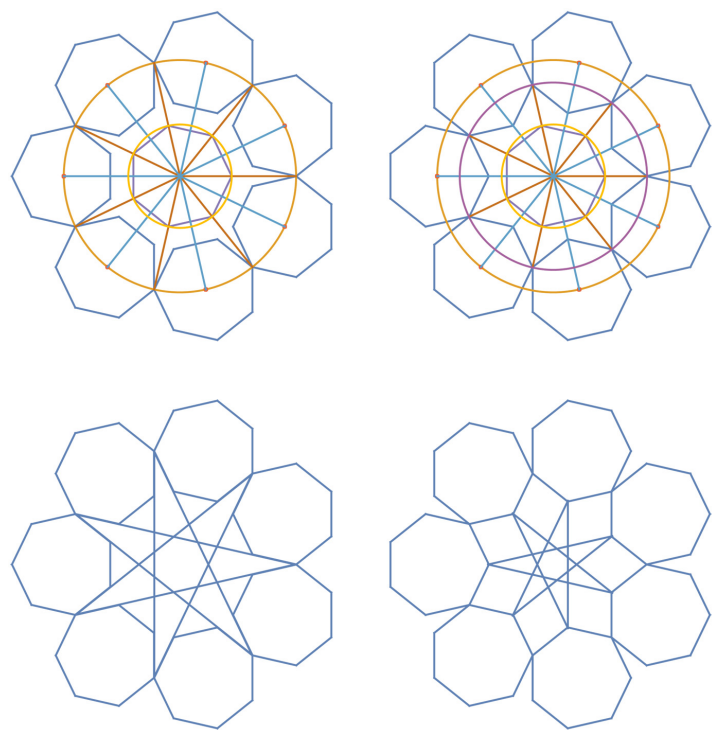

Figure 16. Presteps for Penrose tiles with elements of sevenfold symmetry. The angles at the borders $\frac{3 \pi}{7}$ and $\frac{\pi}{7}$ of the two figures and of the jags of the star-heptagons in the center are dual to each other. They are equal to the inner angles of the jags of the two regular star-heptagons in Figure 5. If the radius of the circumscribed circles to the regular heptagons is set equal to 1 then the distance from the coordinate center to the centers of the regular heptagons in the first generation is equal and is

$R=\frac{\sin \left(\frac{3 \pi}{7}\right)}{\sin \left(\frac{\pi}{7}\right)}=1+2 \cos \left(\frac{2 \pi}{7}\right)=\frac{1}{2 \sin \left(\frac{\pi}{14}\right)}$.
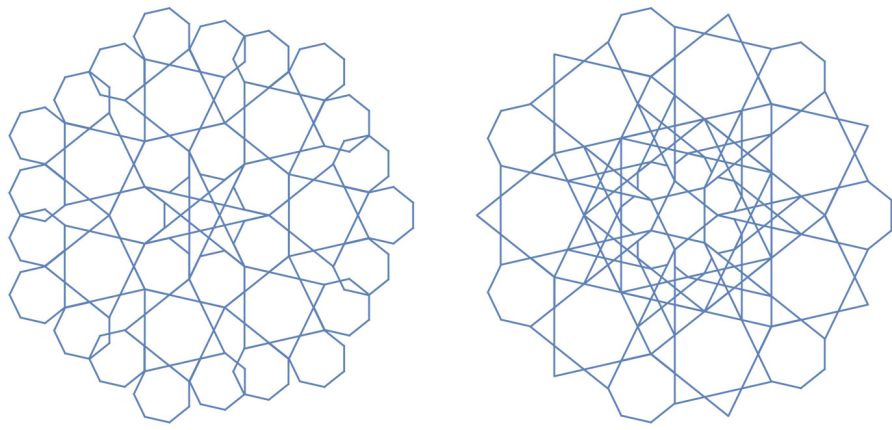

Figure 17. Two basic patterns for Penrose tiles with elements of sevenfold symmetry and with symmetry center. In the first figure we have small overlaps of regular heptagons at the border which can be removed similar to the procedure for parqueting in Figure 11. The inner angle of the jags of regular star-heptagons are $\frac{\pi}{7}$ and $\frac{3 \pi}{7}$ and of the regular heptagon $\frac{5 \pi}{7}$. The outer angles at the border should be equal only to multiples of $\frac{\pi}{7}$. At least, this should be makable with higher rings. The outer angle at the concave points of the whole border is $\frac{\pi}{7}$ in left-hand figure and a star-heptagon of such size as in the center seems to be possible in the next generation. The outer angles at the concave corners in the right-hand figure are $\frac{5 \pi}{7}$ and $\frac{6 \pi}{7}$. 

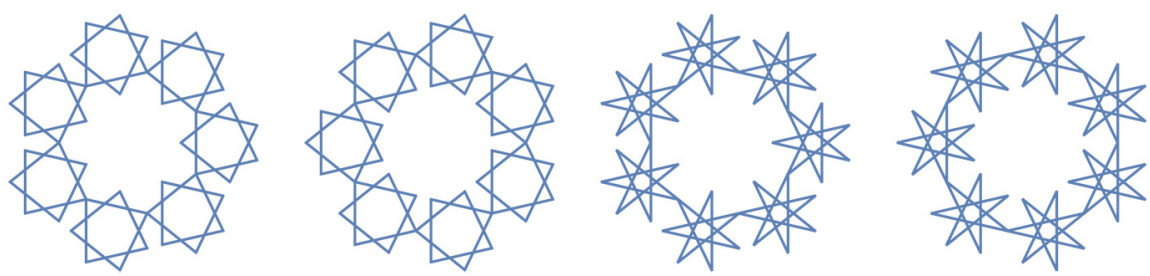

Figure 18. Rings of basic elements of the patterns for Penrose tiles in case of sevenfold symmetry. Patterns of such kind plus analogous ones for regular heptagons where it is possible to change some parameters were generated to make figures such as Figure 17 in "experimental" way by computer. However, Figure 16 was generated by exactly calculating the centers of the regular heptagons.
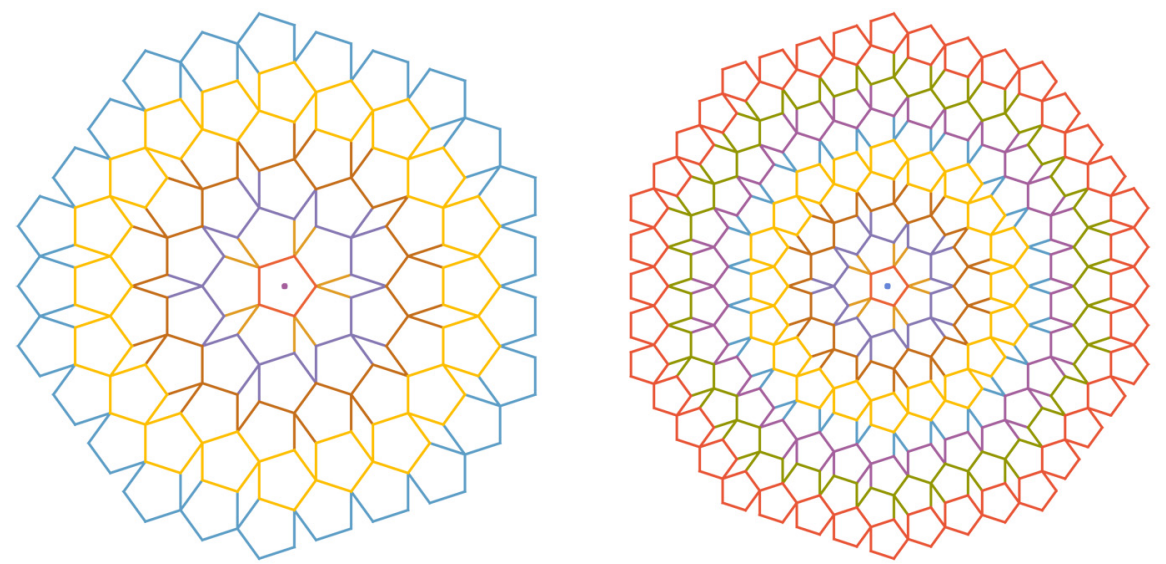

Figure 19. The most simple aperiodic parqueting with symmetry center of five-fold symmetry. The partial figures are analogous to Figure 10 and Figure 14 for seven-fold symmetry with the difference that the small quadrilaterals there are overlaps whereas the small rhombuses here are gaps. We have shown here rings of the fifth and the eighth generation of pentagons. This should be one kind of a Penrose tiling if it is continued to the whole plane.

with basis elements of five-fold symmetry such as regular pentagons plus one additional type of rhombuses. In analogy to seven-fold symmetry in Figure 11 and Figure 12 one may remove the gaps and arrive at (not fully) irregular types of pentagons which cover the whole plane and, clearly, such pictures are known. In most pictures of aperiodic tilings and Penrose tiles the semi-regular pentagon plays a role as an element but Figure 19 should be also an example for aperiodic tiling. One feels that the name "aperiodic" (or "non-periodic") tiling is too general in comparison to "periodic" tiling and the gap between them has to be filled with life that means with further classification and ordering.

\section{Objects with 7-Fold Symmetry in Nature and in Art}

It is believed and true that sevenfold symmetry does not play a great role in geometry. The regular heptagon is the first of the n-gons which cannot be constructed by compass and ruler. The symmetry of the point group $C_{7}$ and $C_{7 v}$ such as the symmetry groups $C_{5}$ and $C_{5 V}$ cannot be extended by translation symmetry to one of the possible lattice symmetries (Bravais lattice). Multiples of the angle 
$\frac{2 \pi}{7} \triangleq 51.429^{\circ}$ and regular heptagons and star-heptagons are not easily to draw without computer.

Seven-fold symmetry in nature and art is possible but in contrast to five-fold symmetry is very rare. In nature we find it in relatively stable form in the flowers of chickweed wintergreen (Trientalis europaea, Trientalis borealis, family Myrsinaceae (myrsine family in the Ericales, formerly positioned within Primulaceae that is in the family of "primroses" with usually fivefold symmetry of the flowers). This is the only example from kingdom of plants known to me. Shubnikov and Koptsik $[15]^{4}$ reproduce in Fig. 29 on p. 28 a ("Lower") animal (a colony of sea squirts Botryllus sp. from ascidians of the Tunicata, phylum Chordata) with sevenfold symmetry from the work of Ernst Haeckel [32] and we found it too $[8]^{5}$. Among the sea stars (or star-“fishes") which possess mostly 5 arms are also a small number of species with sevenfold symmetry as one may see from published pictures.

An example in art is given by D. Sutton in the Section "Perfect Fourteen" pp. 100,101 of [33]. Here we find a picture of a pattern from the mausoleum of Mamluk Sultan Qaytbay in Cairo with a non-perfect (by good will) seven-fold symmetry which contains regular star-heptagons and in the center a regular star-14-gon. It seems that also in Islamic design this is an exception. Although I did not discuss in detail in this paper the constructibility of the regular 17-gon by compass and ruler discovered in young age by Gauss I mention here that in the book of Maor and Jost [3] (pp. 75, 76 with hint to Jost who discovered it) I found to my astonishment an architectural realization of a 17-gon with a photo made in the town of Leipzig which long ago (1955-1961) was the town where I studied physics at the University. It is a 17-sided pattern decorating the floor in a 17-sided glass dome in the "Mädler-Passage" (built in 1912-1914) in the center of the town. I never heard about this during my time in Leipzig.

It seems to be appropriate here to mention the impressive and unparalleled artistic work of Maurits Cornelis Escher who used the symmetry and antisymmetry of figures and the slow transition between them in all shadings playing with light and the transition to hyperbolic and spherical geometry and to spatial geometry in the perspective (e.g., [34] [35], one easily may find other editions). Many authors of the here cited scientific books and mathematical and physical articles liked to include reproductions of the work of Escher. What could have made Escher in his life (1898-1972) from all this by suggestions from Penrose tiles, from seven-fold symmetry and from other novel mathematics by including this in his work?

In many of our figures the color of the objects does not play a role and was chosen more or less incidentally but in some figures I tried to play with color to

${ }^{4}$ I amend here the transcription of the author name "Koptsik" cited in our paper [24].

${ }^{5}$ Due to a photo in a book of Ross Piper (Animal Earth) I have now heavy doubts that it should be mentioned for a species with sevenfold symmetry since it concerns a colony of individuals where this symmetry is by far not stable, even not approximately. 
find a favorable variant.

\section{Conclusions}

In this article, we started from the constructibility of the regular heptagon by bicompasses and ruler that is a pleasure and a delight. Then we considered covering of the plane by regular heptagons that is only possible by overlapping and (or) gaps between a few sorts of tiles obtained from regular heptagons by cut-off of (small) parts and filling gaps that partially lead to beautiful and unusual patterns applicable for parqueting of rotundas and are also a delight. There is a great number of possible variations and only a few principal ones could be shown. The frustration is that their calculation is time-consuming in many cases. The regular heptagon and the possible regular and semi-regular star-heptagons were rarely used up to now for patterns with full or partial seven-fold symmetries. Although it is not possible to make fully periodic tessellations with seven-fold point-group symmetry with some centers for the whole plane it seems to be possible to find analogs of Penrose tiles and also generalizations to color groups and to spherical and hyperbolic geometry seem to be possible. Thus, there is a high potential for future generalization and extension of the shown possibilities of using the regular heptagons for unusual patterns, parqueting, tiling and tessellation.

The next numbers after the 7 which make difficulties in geometry of regular figures are number 9 (trisection of angle $\frac{2 \pi}{3}$ by compassess and ruler is not possible but neusis construction can be applied) and number 11. The number 13 is a little more friendly [8] but the ready form of the construction by bicompasses and ruler is not yet fully clarified.

The frustration with number "Seven" in planar geometry of figures was that many details of their characteristics are troublesome to calculate and to program (though not for principal reason) but errors, to our pleasure, were easily to see when making the drawings by computer. All in all one may say that delight from the results was predominant.

\section{Conflicts of Interest}

The authors declare no conflicts of interest regarding the publication of this paper.

\section{References}

[1] Stillwell, J. (2004) Mathematics and Its History. Second Edition, Springer, New York.

[2] Maor, E. (1998) Trigonometric Delights. Princeton University Press, Princeton and Oxford.

[3] Maor, E. and Jost, E. (2014) Beautiful Geometry. Princeton University Press, Princeton. https://doi.org/10.2307/j.ctt4cgb6n

[4] Conway, J.H. and Guy, R.K. (1996) The Book of Numbers. Springer, New York. 
German Translation: Zahlenzauber, Birkhäuser, Basel. https://doi.org/10.1007/978-1-4612-4072-3

[5] Sutton, A. (2009) Ruler \& Compass Practical Geometric Constructions. Wooden Books, Glastonbury.

[6] Courant, R. and Robbins, H. (1996) What Is Mathematics. Oxford University Press, Oxford. (First Published 1941; I Used the Russian Translation from Prosvyetchenye, Moscow 1967)

[7] Wünsche, A. (2014) Construction of Regular Heptagon by Rhombic Bicompasses and Ruler. Applied Mathematics, 5, 2370-2380.

https://doi.org/10.4236/am.2014.515229

[8] Wünsche, A. (2017) Factorization of Cyclotomic Polynomials with Quadratic Radicals in the Coefficients. Applied Mathematics, 7, 472-506. https://doi.org/10.4236/apm.2017.79032

[9] Weyl, H. (1952) Symmetry. Princeton University Press, Princeton. (Russian Translation, Nauka, Moskva 1963)

[10] Steinhaus, H. (1959) Kaleidoskop der Mathematik. Deutscher Verlag der Wissenschaften, Berlin. In English: Mathematical Snapshots, Oxford Univ. Press, Oxford and Warszawa 1954.

[11] Lyusternik, L.A. (1956) Vypuklyje figury i mnogogranniki (Convex Figures and Polyhedrons). Gostekhizdat, Moskva.

[12] Fejes Tóth, L., Figuren, R. and Teubner, B.G. (1965) Verlagsgesellschaft Leipzig. (Contains Also Spatial Pictures to Consider with Special Red-Green Stereo-Spectacles)

[13] Shubnikov, A.V. (1916) K voprocy o stroyenii kristallov (To the Question about the Structure of Crystals). Isvestija Akademii Nauk, Serija 6, 10, 755-779. (This and Many Other Interesting Articles Are Republished in [14])

[14] Shubnikov, A.V. (1975) Isbrannyje trudy po kristallografii (Selected Works about Crystallography). Nauka, Moskva.

[15] Shubnikov, A.V. and Koptsik, V.A. (1972) Simmetriya v nauke i iskustve (Symmetry in Science and Art; Second Edition, First Edition 1940 According to Preface). Nauka, Moskva.

[16] Koptsik, V.A. (1966) Shubnikovskiye gruppy (Shubnikov Groups), (Its "Contents" Is Translated on p. 4 into English). Izdatelstvo Moskovskovo universitata, Moskva.

[17] Landau, L.D. and Lifshits, E.M. (1982) Electrodynamics of Continuous Media. 2nd Edition, Nauka, Moskva. In English, for Example: Addison-Wesley, Reading, MA, 1987.

[18] Landau, L.D. and Lifshits, E.M. (1989) Quantum Mechanics. Fourth Edition, Nauka, Moskva. In English, for Example: Pergamon Press, New York, 1958.

[19] Dimmock, J.O. and Wheeler, R.G. (1966) Symmetrieeigenschaften magnetischer Kristalle. In: Margenau, H. and Murphy, G.M., Eds., Die Mathematik für Physik und Chemie, Band 2, Chap. 12, D. Van Nostrand Comp., Berlin, 786-832.

[20] Levitin, K.Ye. (1984) Geometricheskaya rapsodia (Geometrical Rhapsody). 2nd Re-

${ }^{6}$ It contains 49 papers of Schubnikov from the 349 mentioned works in the bibliography of the author (among them many encyclopedy articles, book recensions).

${ }^{7}$ From reproductions in this book and the books of $\mathrm{M}$. Gardner translated into Russian and from sources of this kind I became first a little acquainted with name and work of Maurits Cornelis Escher.

${ }^{8}$ This precious book with 723 pages in large format though for a very small group of high specialists is an intellectual achievement but probably very rarely to find in Germany. 
vised Edition, Znaniye, Moskva.

[21] Lyubarskiy, G.Ya. (1958) Teoriya grupp i yeyo primenyeniya v fizike. Fizmatgiz, Moskva. German Transl.: G.J. Ljubarski, Anwendung der Gruppentheorie in der Physik, VEB Deutscher Verlag d. Wiss., Berlin, 1962.

[22] Yale, P.B. (1968) Geometry and Symmetry. Holden-Day, San Francisco. Dover Publ., New York, 1968.

[23] Kleber, W. (1970) An Introduction to Crystallography. VEB Verlag Technik, Berlin.

[24] Wünsche, A. (1971) Optische Gyrotropie als Symmetrieeigenschaft im Rahmen der magnetischen Kristallklassen. Monatsberichte der Deutschen Akademie der Wissenschaften zu Berlin, 13, 480-491. (This Journal of Former GDR Has Ceased Its Publication and, Probably, Is Not Very Propagated)

[25] Coxeter, H.S.M. (1963) Regular Polytopes. Macmillan Company, New York. Dover Publ., New York, 1973.

[26] Grünbaum, B. and Shephard, G.C. (1987) Tilings \& Patterns. W.A. Freeman and Comp., New York. Second Edition: Dover Publ., Mineola (N.Y.) 2016.

[27] Conway, J.H., Burgiel, H. and Goodman-Strauss, Ch. (2008) The Symmetries of Things. A.K. Peters, Wellesley.

[28] Miyazaki, K. (1987) Polyeder und Kosmos. Vieweg, Braunschweig.

[29] Conway, J.H. and Sloane, N.J.A. (1999) Sphere Packings, Lattices and Groups. Springer, New York. https://doi.org/10.1007/978-1-4757-6568-7

[30] Gardner, M. (1997) Penrose Tiles and Trapdoor Ciphers. Revised Edition, Mathematical Association of America, Washington DC.

[31] Conway, J.H. and Smith, D.A. (2003) On Quaternions and Octonions. CRC Press, Boca Raton. https://doi.org/10.1201/9781439864180

[32] Haeckel, E. (2016) Kunstformen der Natur. Kunstformen aus dem Meer. In: Breidbach, O., Ed., Prestel, München, New York. (3. Aufl.). (The Emergence of the Work of Haeckel).

[33] Sutton, D. (2014) Islamic Design. In: Martineaux, J., Ed., Designa (Wooden Books), Bloomsbury, New York, 57-111.

[34] Locher, J.L. (1986) (Herausgeber (Ed.)), Leben und Werk des M.C. Escher. Rheingauer Verlagsgesellschaft.

[35] Ernst, B. (2002) Der Zauberspiegel des M.C. Escher. TASCHEN, Köln. 
Appendix A. Multiples of $\frac{\pi}{14}$ Expressed in Practical Angle Measure

For convenience of the reader we give here Table A1 of the equivalence of angles in natural measure to the practical measure in angle degrees $2 \pi \triangleq 360^{\circ}$

Table A1. Equivalences of angle measures.

\begin{tabular}{|c|c|c|c|c|c|}
\hline$n$ & $\frac{n \pi}{14}$ & Angle degrees & $n$ & $\frac{n \pi}{14}$ & $\begin{array}{c}\text { Angle } \\
\text { degrees }\end{array}$ \\
\hline 1 & 0.22439948 & $12.857^{\circ}$ & 15 & 3.36599213 & $192.857^{\circ}$ \\
\hline 2 & 0.44879895 & $25.714^{\circ}$ & 16 & 3.59039160 & $205.714^{\circ}$ \\
\hline 3 & 0.67319843 & $38.571^{\circ}$ & 17 & 3.81479108 & $218.571^{\circ}$ \\
\hline 4 & 0.89759790 & $51.429^{\circ}$ & 18 & 4.03919055 & $231.429^{\circ}$ \\
\hline 5 & 1.12199738 & $64.286^{\circ}$ & 19 & 4.26359003 & $244.286^{\circ}$ \\
\hline 6 & 1.34639685 & $77.143^{\circ}$ & 20 & 4.48798951 & $257.143^{\circ}$ \\
\hline 7 & $1.57079633=\frac{\pi}{2}$ & $90^{\circ}$ & 21 & $4.71238898=\frac{3 \pi}{2}$ & $270^{\circ}$ \\
\hline 8 & 1.79519580 & $102.857^{\circ}$ & 22 & 4.93678846 & $282.857^{\circ}$ \\
\hline 9 & 2.01959528 & $115.714^{\circ}$ & 23 & 5.16118793 & $295.714^{\circ}$ \\
\hline 10 & 2.24399475 & $128.571^{\circ}$ & 24 & 5.38558741 & $308.571^{\circ}$ \\
\hline 11 & 2.46839423 & $141.429^{\circ}$ & 25 & 5.60998688 & $321.429^{\circ}$ \\
\hline 12 & 2.69279370 & $154.286^{\circ}$ & 26 & 5.83438636 & $334.286^{\circ}$ \\
\hline 13 & 2.91719318 & $167.143^{\circ}$ & 27 & 6.05878583 & $347.143^{\circ}$ \\
\hline 14 & $3.14159265=\pi$ & $180^{\circ}$ & 28 & $6.28318531=2 \pi$ & $360^{\circ}$ \\
\hline
\end{tabular}

Multiples of the angle $\frac{\pi}{14}$ are not the only ones which play a role in our considerations (see, e.g., (8.2) and (8.3)) but they are the most important ones. 